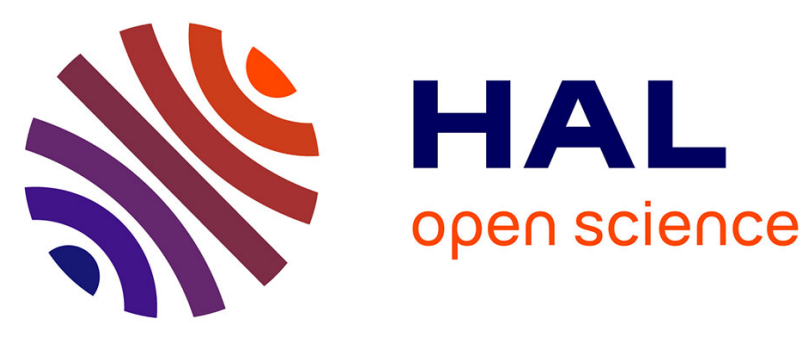

\title{
Virulence of Vibrio harveyi ORM4 towards the European abalone Haliotis tuberculata involves both quorum sensing and a type III secretion system
}

Amandine Morot, Sahar El Fekih, Adeline Bidault, Alizée Le Ferrand, Albane Jouault, Javid Kavousi, Alexis Bazire, Vianney Pichereau, Alain Dufour, Christine Paillard, et al.

\section{To cite this version:}

Amandine Morot, Sahar El Fekih, Adeline Bidault, Alizée Le Ferrand, Albane Jouault, et al.. Virulence of Vibrio harveyi ORM4 towards the European abalone Haliotis tuberculata involves both quorum sensing and a type III secretion system. Environmental Microbiology, 2021, 23 (9), pp.5273-5288. 10.1111/1462-2920.15592 . hal-03454254

\section{HAL Id: hal-03454254 https://hal.science/hal-03454254}

Submitted on 29 Nov 2021

HAL is a multi-disciplinary open access archive for the deposit and dissemination of scientific research documents, whether they are published or not. The documents may come from teaching and research institutions in France or abroad, or from public or private research centers.
L'archive ouverte pluridisciplinaire HAL, est destinée au dépôt et à la diffusion de documents scientifiques de niveau recherche, publiés ou non, émanant des établissements d'enseignement et de recherche français ou étrangers, des laboratoires publics ou privés. 


\title{
Virulence of Vibrio harveyi ORM4 toward the European abalone Haliotis tuberculata involves both quorum sensing and a type III secretion system
}

\author{
Amandine Morot $^{1,2}$, Sahar El Fekih ${ }^{1}$, Adeline Bidault ${ }^{1}$, Alizée Le Ferrand ${ }^{1}$, Albane \\ Jouault ${ }^{2}$, Javid Kavousi ${ }^{1}$, Alexis Bazire², Vianney Pichereau1, Alain Dufour², \\ Christine Paillard ${ }^{1, *}$ and François Delavat ${ }^{1,3, *}$ \\ ${ }^{1}$ Univ Brest, CNRS, IRD, Ifremer, LEMAR, F-29280 Plouzané, France \\ 2Université de Bretagne-Sud, EA 3884, LBCM, IUEM, F-56100 Lorient, France \\ ${ }^{3}$ UMR CNRS 6286 UFIP, University of Nantes, 44000 Nantes, France
}

Keywords : Vibrio harveyi, virulence, Haliotis tuberculata, quorum sensing, type III secretion system, biofilms, LuxO, ExsA

Running Title: Virulence of Vibrio harveyi toward abalones

*Corresponding authors:

francois.delavat@univ-nantes.fr

christine.paillard@univ-brest.fr

This article has been accepted for publication and undergone full peer review but has not been through the copyediting, typesetting, pagination and proofreading process which may lead to differences between this version and the Version of Record. Please cite this article as doi: $10.1111 / 1462-2920.15592$

This article is protected by copyright. All rights reserved. 


\section{Abstract}

Environmental Vibrio strains represent a major threat in aquaculture, but the understanding of their virulence mechanisms heavily relies on the transposition of knowledge from human-pathogen vibrios. Here, the genetic bases of the virulence of Vibrio harveyi ORM4 toward the European abalone Haliotis tuberculata were characterized. We demonstrated that $\operatorname{lux} O$, encoding a major regulator of the quorum sensing system, is crucial for the virulence of this strain, and that its deletion leads to a decrease in swimming motility, biofilm formation, and exopolysaccharide production. Furthermore, the biofilm formation by $V$. harveyi ORM4 was increased by abalone serum, which required LuxO. The absence of LuxO in $V$. harveyi ORM4 yielded opposite phenotypes compared with other Vibrio species including V. campbellii (still frequently named V. harveyi). In addition, we report a full Type III Secretion System (T3SS) gene cluster in the $V$. harveyi ORM4 genome. LuxO was shown to negatively regulate the promoter activity of exsA, encoding the major regulator of the T3SS genes, and the deletion of exsA abolished the virulence of $V$. harveyi ORM4. These results unveil virulence mechanisms set up by this environmentally important bacterial pathogen, and pave the way for a better molecular understanding of the regulation of its pathogenicity.

\section{Significance statement}

Non-human Vibrio pathogens are understudied, and the molecular pathways involved during their infection process frequently rely on knowledge of Vibrio cholerae, hampering new discoveries in the field. In addition, those strains are not necessarily genetically tractable. In this study, we identified key virulence genes in Vibrio harveyi ORM4, a potent pathogen of the abalone Haliotis tuberculata, capable of killing up to $80 \%$ of its host after 3 to 5 days when seawater temperature is permissive. We demonstrated that quorum sensing and a functional Type III Secretion System play a crucial role for the virulence of this strain. Additionally, we showed that the luxO deletion leads to opposite phenotypes when comparing with other Vibrio model species including V. campbellii BAA-1116 (still frequently named $V$. harveyi). This study highlights the necessity to study new Vibrio strains outside already established models. We propose to use V. harveyi ORM4 as a new 
standard for studying the molecular mechanisms involved in the virulence of environmental Vibrio.

This article is protected by copyright. All rights reserved. 


\section{Introduction}

Vibrio-associated diseases cause major economic and ecological plagues worldwide, and are linked to extensive sanitary concerns. Global warming and the associated increase in seawater temperature are thought to amplify these phenomena. Studying Vibrio pathogens is therefore of prime importance in a future attempt to mitigate infections. Particularly, the molecular mechanisms involved during infection need to be better understood, and the key factors have to be deciphered. However, those factors have been most extensively studied in Vibrio models that are human pathogens such as Vibrio cholerae, while non-model (i.e. non-human) pathogens remain far less studied (Le Roux, 2017). Nevertheless, non-model Vibrio represent a major source of pathogenic bacteria in marine environments, causing high mortality both in natural environments and in aquaculture (Paillard et al., 2004; Travers et al., 2015; Ina-Salwany et al., 2019) and the overall economic impact of marine pathogens is estimated in billions dollars yearly (Bondad-Reantaso et al., 2005). Among the pathogens of marine organisms, one can cite Vibrio alginolyticus, which can infect humans but also fishes, clams or shrimps (for review, see Ruwandeepika et al., 2012)), or Vibrio parahaemolyticus, which can also infect shrimps (Vandenberghe et al., 1999). Finally, Vibrio harveyi is notoriously known to infect many economically important marine organisms, including fishes and shrimps (Lee et al., 2002; Austin and Zhang, 2006).

In many bacteria, quorum sensing (QS) systems control collective bacterial behaviours including virulence (Miller and Bassler, 2001). Gene regulation by QS systems is dependent on the concentration of signal molecules called autoinducers, the concentration of which depends on bacterial density. This phenomenon was first described by Nealson et al. (1970) who analysed the regulation of bioluminescence production in Vibrio fischeri. In the Harveyi clade, the QS system of V. harveyi BAA-1116, reclassified as Vibrio campbellii BAA-1116 (Lin et al. 2010), has been largely described, and three types of autoinducers were identified. The binding of autoinducers to their respective histidine kinase receptor feeds a single regulatory signal transduction pathway involving the LuxO protein. At low cell density, the autoinducer concentrations are below the detection threshold of receptors, which induces their autophosphorylation. This autophosphorylation allows the phosphorylation of LuxU, and then of LuxO. Phosphorylated Lux0, together with the alternative sigma factor $\sigma^{54}$, activates the 
transcription of genes encoding small non-coding regulatory RNAs (ncRNAs) (Milton, 2006). These ncRNAs, together with the RNA-binding protein Hfq, inhibit the translation of the major QS regulator gene luxR (Miyamoto et al., 2003; Ball et al., 2017). At high cell density, the autoinducer concentrations reach the detection threshold of receptors, leading to an inhibition of the kinase activities in the phosphorelay. As a consequence, the non-phosphorylated LuxO is unable to induce the expression of the ncRNAs, leading to an increased translation of luxR (Lorenz et al., 2017). The LuxR protein regulates directly or indirectly the expression of up to 625 genes in V. campbellii (van Kessel et al., 2013; Ball and van Kessel, 2019), notably triggering an increased virulence (Noor et al., 2019) and motility (Yang and Defoirdt, 2015), and a decreased biofilm formation (Anetzberger et al., 2009).

Importantly, while this above-described regulatory network is highly conserved among Vibrio (Ng and Bassler, 2009), the downstream genes regulated by the LuxR/HapR family of proteins and the phenotypical outcomes of this gene regulation can greatly differ from species to species (Ng and Bassler, 2009; Boyaci et al., 2016; Ball et al., 2017). Given the recent reclassification of $V$. harveyi BAA-1116 as $V$. campbellii, any conclusion using $V$. campbellii as a reference can lead to over- or mis-interpretation of the QS machinery system and regulation when studying true $V$. harveyi strains. This bias highlights the necessity to establish a new model for a true $V$. harveyi species to decipher the virulence mechanisms of this species. The strain ORM4 used in the present work is a true $V$. harveyi, as shown by pairwise genome comparisons (Table S1).

$V$. harveyi ORM4 is a potent pathogen of the European abalone Haliotis tuberculata, a marine gastropod of high economic value. It was isolated during a severe disease outbreak which resulted in the mortality of 50 to $90 \%$ of the natural European abalone stock in Brittany and Normandy (France) in the late 90's (Nicolas et al., 2002). This strain also provokes massive abalone mortality in laboratory experiments, with between $70 \%$ and $90 \%$ of death occurring within 3 days post-infection (Travers et al., 2008; Cardinaud et al., 2014a). It has been demonstrated that $V$. harveyi ORM4 is causing an immunesuppression in H. tuberculata, by reducing hemocyte activities (Cardinaud et al., 2015). Moreover, a host-centered RNA sequencing approach allowed the de novo assembly and annotation of the transcriptomic profiles of $H$. tuberculata under different conditions, including upon infection by $V$. harveyi ORM4 (Harney et al., 2016). Interestingly, earlier work showed that $V$. harveyi ORM4 is incapable of causing abalone death when the water 
temperature is below $17^{\circ} \mathrm{C}$ or when the abalone is immature, not ready to spawn and not stressed, while an increase of the seawater temperature by only one degree $\left(18^{\circ} \mathrm{C}\right)$ is sufficient to trigger up to $80 \%$ of abalone mortality (Travers et al., 2009a; Cardinaud et al., 2014b). Global climate change and its associated increase in seawater temperature can therefore lead to rapid declines in bioresources, as already observed in Europe for $H$. tuberculata (Huchette and Clavier, 2004) and in the USA, where Haliotis sorenseni and Haliotis cracherodii are officially classified as endangered species (Hobday et al., 2001; Gruenthal and Burton, 2008). Thus, the V. harveyi - H. tuberculata system can be an ideal model to study the effect of global change on the pathogenicity of marine vibrios.

In summary, while knowledge about the response of the abalone upon infection is accumulating, our understanding of the molecular mechanisms of $V$. harveyi ORM4 during infection remains limited. Increasing our knowledge on this environmental strain requires us to set up appropriate genetic tools. The recent development of an efficient electroporation protocol allowed us to rapidly introduce exogenous DNA (replicative plasmids and mini-transposons) within V. harveyi ORM4 (Delavat et al., 2018). Using this procedure, transcriptional fusions have been constructed and introduced into $V$. harveyi ORM4, which showed at the single-cell level that the expression of a flagellar gene is subjected to phenotypic heterogeneity within an isogenic population of $V$. harveyi ORM4 (Delavat et al., 2018). Despite these advances, the key bacterial factors important for abalone infection remain unknown, hampering our understanding of their lifestyle and behaviour during infection.

The objectives of this study were to unveil the genetic bases involved in the virulence of $V$. harveyi ORM4 toward abalone, and to shed a new light on similarities and differences with established knowledge on $V$. campbellii BAA-1116. To reach these objectives, we report here the in vitro and in vivo phenotypic characterizations of both an exsA mutant and a luxO mutant, affected in their type III secretion system and quorum sensing system, respectively. Our results pave the way for a better molecular understanding of the regulation of its pathogenicity in response to climate change.

\section{Experimental procedures}

\section{Strains and culture conditions}

This article is protected by copyright. All rights reserved. 
Unless otherwise stated, Escherichia coli strains were routinely grown at $37^{\circ} \mathrm{C}$ in $\mathrm{LB}$, and $V$. harveyi strains were cultivated at $28^{\circ} \mathrm{C}$ in LBS (LB containing $20 \mathrm{~g} / \mathrm{l} \mathrm{NaCl}$ (final concentration)). If necessary, agar (15 g/l), kanamycin (Km, $100 \mu \mathrm{g} / \mathrm{ml}$ for E. coli, 250 $\mu \mathrm{g} / \mathrm{ml}$ for $V$. harveyi), chloramphenicol ( $\mathrm{Cm}, 5 \mu \mathrm{g} / \mathrm{ml}$ ), trimethoprim (Trim, $10 \mu \mathrm{g} / \mathrm{ml}$ ), ampicillin (Amp, $100 \mu \mathrm{g} / \mathrm{ml}$ ), glucose (Glc, $0.3 \mathrm{~g} / \mathrm{l}$ ), diaminopimelic acid (DAP, $0.3 \mathrm{mM}$ ), Larabinose (L-ara, $0.2 \%$ ), Congo Red $(40 \mu \mathrm{g} / \mathrm{ml})$ or Coomassie Blue $(15 \mu \mathrm{g} / \mathrm{ml})$ were added to the media. Strains and plasmids used in this study are listed in Tables S2 and S3, respectively.

Doubling times of the different Vibrio strains were determined by spectrophotometry. Briefly, one colony of each strain was used to inoculate $5 \mathrm{ml}$ of LBS in tubes, which were incubated for 8 hours at $20^{\circ} \mathrm{C}$ or $28^{\circ} \mathrm{C}$ with shaking. $100 \mu \mathrm{l}$ were subsequently used to inoculate $5 \mathrm{ml}$ LBS in tubes, in triplicates, and tubes were incubated overnight with shaking at their respective temperatures. $50 \mu \mathrm{l}$ of each triplicate were then used to inoculate $100 \mathrm{ml}-$ erlenmeyers containing $20 \mathrm{ml}$ LBS, and OD $_{600 \mathrm{~nm}}$ was measured regularly over 10 hours. Doubling times were calculated from the exponential growth phase of each culture.

\section{Strain constructions and DNA techniques}

Molecular techniques were performed according to standard procedures and by following the recommendations of the reagent suppliers. Targeted chromosomal deletions were performed by double homologous recombination between a suicide plasmid and the chromosome. Briefly, around $800 \mathrm{bp}$ of the upstream and downstream regions of each gene (luxO (HORM4v2_520006) and exsA (HORM4v2_240097)) to be deleted were amplified by PCR (Table S4), and a SOEing PCR (Splicing by Overlap Extension PCR) was performed to fuse the two fragments. The obtained fragment was cloned in the pGEM-T vector (Promega), sequenced to verify the absence of point mutation, and subcloned into pLP12 (Luo et al., 2015), using unique cloning sites. These newly constructed suicide plasmids were transformed in E. coli $\beta$-3914 (Le Roux et al., 2007) carrying a RP4 origin of transfer. Matings were performed by growing the E. coli donor strains (strain $\beta-3914$ carrying each suicide plasmid pFD067 or pFD083), $V$. harveyi ORM4 and, if necessary, an E. coli helper strain carrying the pEVS104 plasmid (Dunn et al., 2006), overnight. The latter strain increases the chance of the suicide plasmid to conjugate from the donor strain to the recipient strain. After overnight growth, $200 \mu \mathrm{l}$ of the E. coli donor strain culture 
were centrifuged, $200 \mu \mathrm{l}$ of the E. coli helper strain were added to the pellet, centrifuged again, and $800 \mu \mathrm{l}$ of $V$. harveyi ORM4 were added to the pellet, before a final centrifugation. The pellet was resuspended with $20 \mu \mathrm{l}$ of a LBS+DAP+Glc solution, which was spotted in a single drop on a $0.2 \mu \mathrm{m}$ acetate filter placed on a LBS+DAP+Glc plate. Matings were allowed to incubate for 24 hours at $28^{\circ} \mathrm{C}$. Filters were subsequently removed, rinsed with $1 \mathrm{ml}$ filter-sterilized seawater (FSSW), and the cell suspension was concentrated by centrifugation and plated on $\mathrm{LBS}+\mathrm{Glc}+\mathrm{Cm}$ plates. After $24 \mathrm{~h}$ to $48 \mathrm{~h}$ incubation, colonies were restreaked on $\mathrm{LBS}+\mathrm{Glc}+\mathrm{Cm}$, and the integration of the suicide plasmid in $V$. harveyi ORM4 was verified by colony-PCR. At least one colony with an integration of the suicide plasmid in the "upstream" region and one in the "downstream" region were grown overnight in $5 \mathrm{ml}$ LBS, before being serially diluted and plated on LBS+L-ara. Colonies obtained after overnight incubation were restreaked on LBS+L-ara and LBS+Cm to control their Cm-sensitivity. The genetic background (revertant wild-type or deletion mutant) of the Cm-sensitive clones was determined by colony-PCR. Deletion mutants were finally stored at $-80^{\circ} \mathrm{C}$ in glycerol stocks.

Complementations of the exs $A$ and $\operatorname{lux} O$ mutants were obtained by amplifying the corresponding genes and their respective promoters, and cloning them into the multicopy plasmid pJLS199 and derivatives. The amplified fragments were cloned in the pGEM$\mathrm{T}$ vector and sequenced to verify the absence of mutations. Fragments were then subcloned (NheI/BamHI digestion) into pJLS199 yielding pFD067 (for the exsA gene) or into pFD077 yielding pFD084 (for the luxO gene). The newly constructed plasmid pFD067 was transferred to the exsA mutant by electroporation, using a recently established protocol (Delavat et al., 2018), but using $\mathrm{MgCl}_{2}, 6 \mathrm{H}_{2} \mathrm{O}$ instead of $\mathrm{MgCl}_{2}$. pFD084 was transferred by tri-parental mating, using strains $V$. harveyi ORM4 $\Delta l u x O$ and two E. coli strains carrying pEVS104 and pFD084, respectively. Complemented mutants were stored at $-80^{\circ} \mathrm{C}$ in a glycerol stock, and trimethoprim was always used to maintain the plasmid in these strains.

The IuxO and T3SS annotated genomic regions of $V$. harveyi ORM4, and genes HORM4_520123 and HORM4_520124 were deposited in the European Nucleotide Archive (ENA, https://www.ebi.ac.uk/ena/browser/home) under the accession numbers LR992915, LR992914, HG993337 and HG993338, respectively.

mRNA assay by reverse transcription-quantitative PCR (RT-qPCR)

This article is protected by copyright. All rights reserved. 
RNA extractions were performed from LBS-grown cultures. One colony of $V$. harveyi ORM4, the $\triangle$ exs $A$ and $\triangle l u x O$ strains were used to inoculate $5 \mathrm{ml}$ of LBS in tubes and incubated for $8 \mathrm{~h}$ at $20^{\circ} \mathrm{C}$ with shaking. $100 \mu \mathrm{l}$ of each culture were used to inoculate in triplicate $5 \mathrm{ml}$ LBS and tubes were incubated overnight at $20^{\circ} \mathrm{C}$ with shaking. The next morning, $50 \mu \mathrm{l}$ were used to inoculate $20 \mathrm{ml}$ LBS (in $100 \mathrm{ml}$ erlenmeyer). Cultures were harvested at $\mathrm{OD}_{600 \mathrm{~nm}} 0.1$ centrifuged, and pellets were subsequently resuspended in the same volume of LBS. Tubes were incubated at $20^{\circ} \mathrm{C}$ with shaking for $1 \mathrm{~h}$. After incubation, tubes were centrifuged for $10 \mathrm{~min}$ at $4000 \mathrm{~g}$ at $4^{\circ} \mathrm{C}$, and the supernatant was discarded. Total RNA were extracted with TRI reagent according to the manufacturer's procedure (Sigma-Aldrich). Residual DNA were eliminated using the Turbo DNAse (Thermo Fisher Scientific). $1 \mu \mathrm{g}$ of RNA were converted to single-stranded cDNA using the RevertAid H Minus First Strand cDNA Synthesis kit (Thermo Fisher Scientific) according to the manufacturer's instructions. The concentration and quality of RNA before and after DNAse treatment were respectively measured with a fluorometer (Qubit 4, Thermo Fisher Scientific) and by electrophoresis (Bioanalyzer Agilent 2100). mRNA of interest were quantified by real-time PCR of their cDNA. Each primer pair used to amplify within HORM4v2_240099, HORM4v2_240127 and HORM4v2_1190007 (Table S4) were validated by verifying that the PCR efficiency (E) was above 0.95 . The $15 \mu \mathrm{l}$ reactions contained 7.5 $\mu \mathrm{l}$ of $2 \mathrm{X}$ SYBR Green I Master Mix, $0.1 \mu \mathrm{g}$ of cDNA and $0.3 \mu \mathrm{M}$ of each primer. The conditions were $95^{\circ} \mathrm{C}$ for $7 \mathrm{~min}$ and 40 cycles at $95^{\circ} \mathrm{C}$ for $10 \mathrm{~s}, 60^{\circ} \mathrm{C}$ for $20 \mathrm{~s}$ and $72^{\circ} \mathrm{C}$ for $20 \mathrm{~s}$. PCR reactions were performed in triplicate (LightCycler 480 Instrument II, Roche Life Sciences). The relative quantification of mRNA was obtained by the comparative CT

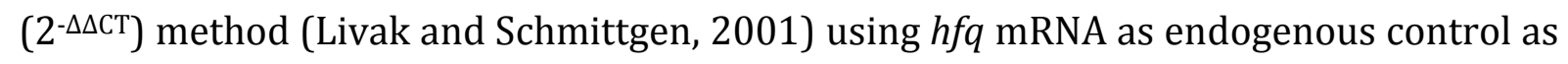
previously reported (Waters et al., 2010).

\section{Motility}

$V$. harveyi strains were grown overnight in LBS at $20^{\circ} \mathrm{C}$ or $28^{\circ} \mathrm{C}$ and diluted to a concentration of $5 \times 10^{8} \mathrm{CFU} / \mathrm{ml}$ (Travers et al., 2008). $2 \mu \mathrm{l}$ of this concentration-adjusted suspension were plated on LBS containing $0.2 \%$ agar, or on "poor motility plate" $(0.2 \%$ agar, $1 \%$ tryptone, $2 \% \mathrm{NaCl}$ and $35 \mathrm{mM} \mathrm{MgSO}_{4}$ ) (Tischler et al., 2018). Halo diameters were measured regularly.

The complemented exsA and luxO mutants were grown in LBS+Trim and compared with $V$. harveyi ORM4 containing the empty pFD085 plasmid. After 10 hours of incubation at 
their respective temperature, the halo diameters of each strain were recorded. Every strain was tested at least in triplicate.

\section{Biofilm production}

Biofilm production was measured from cultures grown in LBS+Trim or in serum+Trim. Serum was prepared by pipetting abalone haemolymph with a $2.5 \mathrm{ml}$ syringe (Terumo) and a needle (Terumo, 25G, 0.5x16 mm). Haemolymph was centrifuged for 5 minutes at $1150 \mathrm{~g}$ at $4^{\circ} \mathrm{C}$, and supernatant was filter-sterilized using a $0.22 \mu \mathrm{m}$ filter to obtain the serum (kept on ice). Strains carrying either pFD085 (empty plasmid) or the plasmid used for complementation were grown overnight in $\mathrm{LBS}+\mathrm{Trim}$ at $20^{\circ} \mathrm{C}$ or $28^{\circ} \mathrm{C}$. Every suspension was diluted with LBS+Trim to $\mathrm{OD}_{590 \mathrm{~nm}} 0.1$, and aliquots of $200 \mu \mathrm{l}$ were transferred to individual wells (in quadruplicate) of a 100-well polystyrene microplate (Bioscreen plates Honeycomb, Thermo Scientific) for the production of biofilms in LBS+Trim. For biofilm production in serum, $900 \mu \mathrm{l}$ of this OD-adjusted suspension were centrifuged, and pellets were resuspended with $900 \mu \mathrm{l}$ serum+Trim, before $200 \mu \mathrm{l}$ were aliquoted in the microplate (in quadruplicate). Microplates were placed in a Bioscreen microplate reader (Labsystems) without shaking at $20^{\circ} \mathrm{C}$ or $28^{\circ} \mathrm{C}$, and the $0 D_{590 n m}$ was measured every 10 minutes. After 48 hours, cell suspensions were removed by inversion, and $220 \mu \mathrm{l}$ of a Crystal Violet (CV, $0.1 \%$ ) solution were added in every well and left for 10 minutes. Wells were washed twice with distilled water and the microplate was left for drying at room temperature for 24 hours. CV was dissolved by adding $30 \%$ acetic acid for 10 minutes, and OD590nm was measured. The absorbance measured in every well was normalized, by dividing the OD590nm measured after crystal violet staining by the maximal OD $590 \mathrm{~nm}$ measured during the growing time of the corresponding well.

\section{Polysaccharide production}

Overnight cultures of strains carrying either pFD085 (empty plasmid) or pFD084 (complementation of luxO) were diluted 1:100 in fresh LBS+Trim medium, and exponentially-growing suspensions were diluted to an $0 D_{600 \mathrm{~nm}}$ at $0.2 .10 \mu \mathrm{l}$ of these ODadjusted suspensions were spotted on LBS plates containing Congo Red and Coomassie Blue (Bassis and Visick, 2010). Plates were incubated for 24 hours, before the colour of each spot was observed by eyes and pictures were acquired. The red/brown-shifted colour intensity of the spot is used as a proxy for EPS production (Bassis and Visick, 2010). 


\section{Fluorescence microscopy}

Strains V. harveyi ORM4 and V. harveyi ORM4 $\Delta l u x O$, carrying the $\mathrm{P}_{\text {exsA-gfp transcriptional }}$ fusion from the pFD090 plasmid were grown for 8 hours in $5 \mathrm{ml} \mathrm{LBS+Trim}$, and $100 \mu \mathrm{l}$ were used to inoculate $5 \mathrm{ml}$ LBS+Trim in tubes, in triplicates. The next morning, $5 \mu \mathrm{l}$ were used to inoculate $5 \mathrm{ml}$ LBS+Trim in tubes (1/1000 dilution). At least 5 pictures per replicate were acquired from the overnight cultures, and after 3, 6 and 9 hours of growth, using the Zeiss Axio Imager.M2 microscope equipped with a 100X Apochromat oil objective, at 200 ms exposure time (with 25\% LED intensity). Fluorescence of single cells was quantified using an in-house written Matlab script (Delavat et al., 2016), with at least 1209 cells per condition.

\section{Infection experiments}

Mature abalones $(6-7 \mathrm{~cm})$ were brought from France Haliotis (Plouguerneau, France) and left in a $1 \mathrm{~m}^{3}$-tank with circulating seawater $\left(19.5^{\circ} \mathrm{C}+/-1^{\circ} \mathrm{C}\right)$. After 2 weeks of acclimation, 12 abalones per condition (in triplicate) were placed in smaller tanks containing 10 liters seawater at the same temperature, with a bubbling system. Tanks were infected for 24 hours by balneation with $5 \times 10^{6} \mathrm{CFU} / \mathrm{ml}$ (Travers et al., 2008) of $V$. harveyi ORM4, of the different deletion mutants of $V$. harveyi ORM4 or of $V$. harveyi LMG 7890, an abalone-avirulent strain (Travers et al., 2009b), all grown overnight in LBS. Balneation allows reproduction of the natural route of animal infection, since bacteria are inoculated into seawater and are not injected directly in the animal. For the second infection experiment using the complemented mutants, all strains carried either the empty plasmid pFD085 or the plasmid used for complementation and were all grown overnight in LBS+trimethoprim. Seawater was renewed every 24 hours in every tank, and dead abalones were counted and removed daily.

\section{Statistical analyses}

All statistical analyses were performed using GraphPad Prism. P values $<0.05$ were considered statistically significant.

\section{RESULTS}

This article is protected by copyright. All rights reserved. 
The quorum sensing LuxO regulator does not affect the growth characteristics of $\mathrm{V}$. harveyi ORM4

To unveil the role(s) of the QS system of $V$. harveyi ORM4, we inactivated the luxO gene, encoding the homolog of a major regulatory protein described extensively in $V$. campbellii BAA-1116. To obtain a $\triangle l u x O$ mutant, we constructed the pFD083 suicide plasmid using the pLP12 vector, which has originally been designed for Vibrio strains and relies on the L-arabinose-inducible Vmi480 toxin to force the second recombination event and the excision of the cointegrate (Luo et al., 2015). The pFD083 suicide plasmid, containing around $800 \mathrm{bp}$ of each of the two regions flanking the $\operatorname{lux} O$ gene, could be integrated into the genome of $V$. harveyi ORM4, as observed by the growth of chloramphenicol-resistant colonies and by dedicated colony-PCR. Induction of Vmi480 led to the excision of the vector from the genome, ultimately leading to the generation of a $\mathrm{V}$. harveyi ORM4 $\triangle l u x O$ deletion mutant in 7 out of the 15 clones tested by PCR.

$V$. harveyi ORM4 grew optimally at $28^{\circ} \mathrm{C}$ in LBS, displaying a doubling time of $36.5 \pm 1.9$ min (Fig. 1), similarly (two-tailed t-test, $\mathrm{p}=0.443$ ) to the $\Delta l u x O$ mutant $(35.4 \pm 1.3 \mathrm{~min}$ at $28^{\circ} \mathrm{C}$ ). A decrease in temperature to $20^{\circ} \mathrm{C}$ (permissive temperature for abalone infection (Travers et al., 2009a)) led to an about 1.9-fold longer doubling time, both for $V$. harveyi ORM4 and for $V$. harveyi ORM4 $\Delta l u x O(65.7 \pm 1.9 \mathrm{~min}$ and $68.7 \pm 0.7 \mathrm{~min}$, respectively). Thus, despite the supposed pleiotropic effect of the QS system (van Kessel et al., 2013), the absence of LuxO did not influence significantly the doubling time of $V$. harveyi ORM4 under these conditions. Moreover, a complementation of $\operatorname{lux} O$ in the $\Delta \operatorname{lux} O$ mutant, through the use of the multi-copy plasmid pFD084, did not impair the growth phenotype (Fig. S1).

The luxO mutant showed an altered motility capacity

Bacteria from the genus Vibrio are frequently motile, and this swimming capacity could play a role during infection, by allowing the bacteria to swim toward their host and colonize it. Therefore, we tested whether V. harveyi ORM4 is motile, and whether the QS system regulates, via LuxO, the motility in this strain. Using $0.2 \%$ agar plates, we could demonstrate that the wild type strain was highly motile, both on rich (LBS) and "poor" plates, with a higher motility level on "poor" plate (Fig. 2). As expected, motility was 
higher on both plates at $28^{\circ} \mathrm{C}$, the optimal growth temperature of $V$. harveyi ORM4, as compared to the motility at $20^{\circ} \mathrm{C}$. Interestingly, the $\Delta l u x O$ mutant was impaired in motility, with 1.5 to 2 -fold decrease in the swimming diameters under every tested condition.

We subsequently complemented the deletion of luxO by introducing in trans the functional $\operatorname{lux} O$ gene and its own promoter on the pFD084 plasmid. Surprisingly, the standard electroporation protocol recently developed for $V$. harveyi ORM4 and other marine bacterial strains (Delavat et al., 2018) was inefficient for the $\Delta l u x O$ mutant, despite repeated attempts. The plasmid was therefore introduced into the $\Delta l u x O$ strain by triparental mating. This complementation restored the motility phenotype to the wild type level, at both temperatures on the two media (Fig. S2). Therefore, an intact QS system is required for full swimming motility of $V$. harveyi ORM4.

The luxO mutant has an impaired biofilm formation capacity

Since biofilm formation could be important for the infection process and is likely QSdependent, biofilms of $V$. harveyi ORM4 and $V$. harveyi ORM4 $\triangle l u x O$ were grown in microtiter plates without shaking at $20^{\circ} \mathrm{C}$ and $28^{\circ} \mathrm{C}$, and the biofilm amounts were quantified. V. harveyi ORM4 was able to form biofilms when grown in LBS, irrespective of the incubation temperature (Fig. 3), and this biofilm was mostly located at the bottom of the wells. Interestingly, the biofilm biomasses increased 5 to 10 -fold when cells where incubated in the presence of serum from abalone haemolymph, as compared to the one on LBS medium (Fig. 3E and 3F). This suggests that the serum somehow induces biofilm formation in $V$. harveyi ORM4. This induction of biofilm formation seems to be strainspecific, because biofilm production was not induced by serum when using $V$. harveyi LMG 7890, an abalone-avirulent strain (Nicolas et al., 2002). V. harveyi ORM4 $\Delta l u x O$ showed a 2 to 3-fold decrease in the amount of biofilm formed in LBS and a 10-200 fold decrease in serum (Fig. 3C and 3D), compared to V. harveyi ORM4 (Fig. 3A and 3B). Moreover, this mutant failed to produce more biofilm in the presence of serum (Fig. 3E and 3F), suggesting that the induction of biofilm formation in abalone serum is QS-dependent. As expected, a functional complementation in the $\Delta l u x O$ mutant restored the production of biofilm and its induction in the presence of serum (Fig. S3).

This article is protected by copyright. All rights reserved. 
Since exopolysaccharides (EPS) are major components of biofilms matrixes (Casillo et al., 2018), we evaluated the EPS production of $V$. harveyi ORM4, the $\Delta l u x O$ mutant and the luxO complemented mutant strains by spotting them onto LBS plates containing the Congo Red dye, which binds polysaccharide-containing structures. After $24 \mathrm{~h}$ of incubation, the spots corresponding to $V$. harveyi ORM4 showed a reddish colour and a red ring close to the extremity of the spot, indicating the presence of some EPS. On the same plate, the spot of the $\Delta l u x O$ mutant is much paler and the reddish ring is basically absent, while complementing the deletion of $\operatorname{lux} O$ restored the wild type phenotype (Fig. S4). The reduced EPS production by the $\triangle l u x O$ mutant could be at least in part responsible for its decreased biofilm formation ability.

\section{LuxO is crucial for virulence of $\mathrm{V}$. harveyi ORM4}

The decreased motility (Fig. 2) and biofilm formation capacities (Fig. 3) displayed by the $\Delta l u x O$ mutant prompted us to examine whether the pathogenic properties are also affected in this mutant. To address this question, mature abalones $(6-7 \mathrm{~cm})$ were infected by balneation with $V$. harveyi ORM4, V. harveyi LMG 7890, or V. harveyi ORM4 $\Delta l u x O$, and the number of surviving abalones was counted every day. $V$. harveyi ORM4 triggered rapid mortality, since only $44.4 \pm 19.2 \%$ of abalones survived infection after 5 days, and only $25 \pm 8.3 \% 5$ days later (Fig. 4A). On the other hand, infection with $V$. harveyi LMG 7890 did not result in any abalone mortality, with $100 \%$ of survival after 10 days (Fig. 4A). When using $V$. harveyi ORM4 $\triangle l u x O$, the mortality of abalones was abolished, since $95.8 \pm 5.9 \%$ of abalones survived after 10 days of balneation (Fig. 4A). Thus, LuxO, and therefore a fully functional QS system, seems to be essential to the virulence of this strain.

Because variability between abalone killing may occur from experiment to experiment, we repeated the infection experiment, and included the $V$. harveyi ORM4 $\Delta l u x O$ mutant complemented with the wild-type $\operatorname{lux} O$ gene. This second infection experiment confirmed our previous findings, i.e. that V. harveyi ORM4 is a potent pathogen, that the abalones used in this second experiment were still sensitive to vibriosis, and that the deletion of luxO led to an impaired virulence of $V$. harveyi ORM4 (Fig. 4B). Moreover, the complementation restored the wild type virulent phenotype, with $13.9 \pm 4.8 \%$ of survivors after 10 days (two-way ANOVA at day 10, p=0.1831 in comparison with the wild type). Host death was furthermore accelerated when using the complemented mutant, since 
only $22.2 \pm 4.8 \%$ of abalones survived after 5 days, compared with $50 \pm 8.3 \%$ for the wild type $(\mathrm{p}<0.0001)$. This could be due to an overexpression of the luxO gene since the latter is carried by a multi-copy plasmid in the complemented strain. These results confirmed that the observed increase in survival rate was due to the luxO deletion. Thus, LuxO plays a key role in the regulation of the molecular mechanism(s) involved directly or indirectly in infection of the European abalone H. tuberculata by $V$. harveyi ORM4.

V. harveyi ORM4 harbours genes for a complete Type III Secretion System

An expert annotation of the genome sequence of $V$. harveyi ORM4 led to the identification of a 33-kb genetic region encoding a putative T3SS. This region is very similar to the region encoding the T3SS1 in V. parahaemolyticus strain RIMD2210633, responsible for the cytotoxicity in host cells during infection (Zhou et al., 2010) (Fig. 5 and Table S5). All genes encoding the structural proteins and the regulators ExsA (HORM4v2_240097), ExsC (HORM4v2_240092), ExsD (HORM4v2_240098) and ExsE (HORM4v2_240091) are found in this region, suggesting a functional secretion system in $V$. harveyi ORM4 (Table S5).

Importantly, we identified in the genome of $V$. harveyi ORM4 orthologs for VopQ (HORM4v2_240119) and VPA0450 (HORM4v2_520123, which lies outside of the T3SS gene cluster), with $82.72 \%$ and $89.74 \%$ of amino acid identity, respectively. VopQ and VPA0450 are two of the four proteins known to be secreted by the T3SS1 in $V$. parahaemolyticus (VopQ, VPA0450, VopR and VopS). VopQ affects lysosomal homeostasis by deacidification (Sreelatha et al., 2013) in V. parahaemolyticus, while VPA0450 is an inositol polyphosphatase that destabilizes plasma membranes (Broberg et al., 2010). The translocation of VPA0450 requires VPA0451, a cargo chaperone protein (Waddell et al., 2014) for which an ortholog was found in V. harveyi ORM4 outside the T3SS region and next to the gene for VPA0450 (HORM4v2_520124).

In close vicinity to $v o p Q$, a large ORF (963 codons) encoding an arginine ADPribosyltransferase was furthermore identified in $V$. harveyi strain ORM4 (HORM4v2_240111). Once translocated in eucaryotic cells, the ADP-ribosyltransferase of $V$. cholerae catalyses the transfer of ADP-ribose to guanine nucleotide regulatory $(G)$ proteins, ultimately leading to increased cAMP level, efflux of chloride ions into the lumen, causing diarrhea (for a review, see (Herrera and Satchell, 2020)). This protein, possibly 
secreted by the T3SS of $V$. harveyi ORM4, may play a similar role in the virulence of this strain, and would deserve further studies.

\section{LuxO regulates the expression of the T3SS}

To assess a possible link between LuxO and the T3SS of $V$. harveyi ORM4, the promoter activity of the exsA gene, encoding the major activator of the T3SS genes, was quantified by epifluorescence microscopy at the single cell level. This was performed by following the expression of a $\mathrm{P}_{\text {exsA }}-g f p$ transcriptional fusion, expressed from the pFD090 plasmid introduced in $V$. harveyi ORM4 and $V$. harveyi ORM4 $\Delta l u x O$, and by quantifying the GFP activity in single cells for both genetic backgrounds. We could demonstrate that exsA is expressed under in vitro condition in $V$. harveyi ORM4, as revealed by the fluorescence emission in this genetic background (Fig. 6B). Moreover, its expression was higher in early exponential phase, when cells were at low cell-density (Fig. 6A, one-way ANOVA with Tukey test, comparing time 3 hours with the other time points, $p<0.0001$ ). Importantly, these results also showed that the $\mathrm{P}_{\text {exsA-gfp }}$ reporter gene was more expressed in the $\triangle l u x O$ mutant, at all time points (Fig 6A), demonstrating that the $\mathrm{QS}$ system regulates the expression of the exsA gene. In addition, RT-qPCR experiments showed that the core T3SS genes HORM4v2_240099 and HORM4v2_240127 are both overexpressed in the $\triangle \mathrm{luxO}$ mutant (Fig. 6C), demonstrating the T3SS regulation by QS, either directly or indirectly, in V. harveyi ORM4 .

The T3SS-regulator ExsA is essential for the virulence of V. harveyi ORM4

Since LuxO plays a key role in the virulence of $V$. harveyi ORM4 toward abalones and regulates the expression of the T3SS via exsA, the exsA gene has been deleted in strain $V$. harveyi ORM4. We first demonstrated by RT-qPCR experiments that ExsA regulates genes of the T3SS, since HORM4v2_240099, encoding a protein from the YscB family involved in preventing the secretion of effectors through the T3SS (Plano and Schesser, 2013), is induced in the $\Delta$ exs A mutant, while the T3SS ATPase (HORM4v2_240127) is repressed (Fig. 6C).

Importantly, apart from the inactivation of the T3SS, the $\triangle$ exs $A$ mutant showed physiological characteristics which are similar to those of the wild type $V$. harveyi ORM4. 
Indeed, its doubling time $\left(67.3 \pm 1.6 \mathrm{~min}\right.$ at $20^{\circ} \mathrm{C}$ and $34.7 \pm 1.1 \mathrm{~min}$ at $\left.28^{\circ} \mathrm{C}\right)$ appeared indistinguishable from that of the wild type (Fig. S5), its motility was similar to the motility of the wild type (Fig. S6), and this mutant was not impaired in biofilm formation (Fig. S7).

However, when abalones were infected with $V$. harveyi ORM4 $\triangle$ exs $A$, the mortality was abolished, as no abalone had died after 10 days, a result identical to non-infected abalones (two-way ANOVA, p>0.9999, Fig. 7A). This experiment thus demonstrated that the T3SS is essential for $V$. harveyi ORM4 to induce mortality in abalones. To confirm this result, we complemented the deletion of exs $A$ in $V$. harveyi ORM4 by transferring the pFD067 plasmid, containing the exsA gene and its own promoter, in $V$. harveyi ORM4 $\triangle$ exsA. Complementing the deletion of exs $A$ restored the virulence of $V$. harveyi ORM4 $\triangle$ exs $A$, since $55.6 \pm 19.2 \%$ of abalones survived after 5 days (two-way ANOVA, p=0.9188 comparing with $V$. harveyi ORM4) and 33.3 $\pm 22 \%(\mathrm{p}=0.7726)$ after 10 days (Fig. 7B).

Taken together, these experiments demonstrated that the T3SS of $V$. harveyi ORM4 is essential for the virulence of this strain.

\section{Discussion}

Although $V$. harveyi is a major marine pathogen, the mechanistical details involved in the virulence of true $V$. harveyi strains are scarce since the best-known strain within this species, $V$. harveyi BAA-1116, has been reclassified within the $V$. campbellii species (Lin et al., 2010). In this study, we aimed at filling this gap, by deploying a dual genetic and functional approach to assess the role of potential major molecular regulators of virulence in $V$. harveyi ORM4, which is a true $V$. harveyi strain (Table S1). We first focused on luxO, encoding a major regulator of the QS system (Miyamoto et al., 2003), regulating the translation of LuxR and ultimately modulating the expression of up to 625 genes in $V$. campbellii (van Kessel et al., 2013; Ball and van Kessel, 2019).

In strains belonging to various species from the genus Vibrio, including V. campbellii BAA1116 (Tu and Bassler, 2007), V. cholerae like El Tor strain C6706 (Ng and Bassler, 2009), V. fischeri strains (Miyashiro and Ruby, 2012) and V. parahaemolyticus BB22 (GodePotratz and McCarter, 2011), a luxO deletion locks the cells in a "high cell-density"-like state, with an increased production of LuxR. Given the conserved QS network architecture, it is widely accepted that it is conserved among Vibrionaceae. The following 
discussion will therefore refer to the physiological state of the luxO mutant of ORM4 as a "high cell-density" -like state. A thorough future study will be however necessary to confirm this hypothesis, by the construction and physiological characterization of various mutants including, but not restricted to, luxR.

In this study, we deleted the luxO gene of $V$. harveyi ORM4, and the mutant was physiologically characterized. Despite the supposedly pleiotropic effect of LuxO, the deletion of its gene did not affect the growth rate of $V$. harveyi ORM4 under in vitro conditions, and the mutant as well as the wild type strain grew faster at $28^{\circ} \mathrm{C}$ than at $20^{\circ} \mathrm{C}$ (Fig. 1). This absence of difference between $V$. harveyi ORM4 and the $\Delta l u x O$ mutant is rather surprising, since a mutant of $V$. campbellii BAA-1116 in which the luxO gene has been inactivated by a Tn5 transposon grows twice slower than the parental strain (Nackerdien et al., 2008). This difference might be due to the fact that V. campbellii BAA1116 is a luminescent bacterium, and that locking cells in a "high cell-density"-like state by inactivating $\operatorname{luxO}$ leads to a constitutive overproduction of luminescence, which is energetically costly for the cells. However, it might also be because V. campbellii BAA1116 is genetically and physiologically different from $V$. harveyi ORM4, and so might be their QS.

In contrast to the absence of growth phenotype, $V$. harveyi ORM4 $\triangle l u x O$ shows a clear decrease in motility, as compared to the wild type motility measured on swimming plates both at 20 and $28^{\circ} \mathrm{C} \mathrm{(Fig.} \mathrm{2).} \mathrm{It} \mathrm{should} \mathrm{be} \mathrm{noted} \mathrm{that} \mathrm{motility} \mathrm{was} \mathrm{not} \mathrm{completely} \mathrm{abolished}$ in the $\triangle \operatorname{luxO}$ mutant, which could be explained by the presence of lateral flagella in $V$. harveyi, which is mainly responsible for swimming (Montanchez and Kaberdin, 2020). This observation is opposite to what is known for $V$. campbellii BAA-1116, where locking cells in a "high cell-density"-like state (by the D47A mutation in Lux0) leads to a significant increase in motility compared with the motility of cells producing the wild type Lux0, while a constitutively inactive QS (by the D47E mutation in LuxO) leads to a decreased motility (Yang and Defoirdt, 2015). Thus, LuxO seems to have opposite effects on motility in $V$. harveyi ORM4 and $V$. campbellii BAA-1116.

Of note, "temperature" and "quantity of nutrients" are two important factors for $V$. harveyi ORM4 motility, since cells swim faster at $28^{\circ} \mathrm{C}$ than at $20^{\circ} \mathrm{C}$, and twice faster on a "poor plate" than on a rich plate (Fig. 2). This can be explained by the necessity for cells to prospect for nutrients on the "poor plate" experiment (Adler, 1966). 
Motility and biofilm formation are tightly linked. Indeed, work on $V$. cholerae showed that mutants impaired in motility also have a decreased capacity in biofilm formation (Watnick and Kolter, 1999), and that blocking flagellar rotation leads to an inhibition of expression of the vps cluster involved in Vibrio polysaccharide production (Lauriano et al., 2004). In addition, biofilm production can be an important process for host colonization. Indeed, $V$. fischeri colonization of its squid host Euprymna scolopes is dependent on the symbiosis polysaccharide (syp) gene locus, the expression of which leads to biofilm formation (Yip et al., 2005). In our study, we demonstrated that $V$. harveyi ORM4 is able to form biofilms, and that the biofilm amount does not change, whether cells were grown at 20 or $28^{\circ} \mathrm{C}$ (Fig. 3). Interestingly, this biofilm formation is dependent on an intact QS system, since the $\triangle l u x O$ mutant forms much less biofilm (Fig. 3) and less EPS (Fig. S4) as compared to the wild type. It means that locking cells of V. harveyi ORM4 in a "high cell-density"-like state (in the $\Delta l u x O$ mutant) is detrimental to biofilm formation. This observation is similar to what is known from V. compbellii BAA-1116. Indeed, a luxO deletion in BAA-1116 leads to a decrease in biofilm production, and this decrease is linked with the generation of a more homogeneous population, highlighting that phenotypic heterogeneity is important for biofilm formation (Anetzberger et al., 2009). In addition, a $\Delta l u x O$ mutant of $V$. cholerae also leads to a decreased biofilm production (Zhu et al., 2002). This is due to the overproduction of HapR (the LuxR homolog in V. cholerae) in this mutant strain, which is a repressor of the Vibrio polysaccharide synthesis (vps) operons (Zhu and Mekalanos, 2003).

Interestingly, the quantity of biofilm formed by $V$. harveyi ORM4 is 5 to 10 -fold higher when cells grew in the presence of serum from abalone haemolymph as compared to the quantity of biofilm formed in LBS (Fig. 3). Moreover, this induction by abalone serum is abolished in the $V$. harveyi ORM4 $\Delta l u x O$ mutant. This suggests that LuxO is activated, either through a signaling molecule found in serum or through a yet-unknown mechanism, and this activation is relayed via the QS system to the biofilm production regulatory machinery. However, we also demonstrated that serum induction of biofilm formation is absent in V. harveyi LMG 7890, an abalone-avirulent strain. This would suggest that the induction of biofilm formation by molecules found in the abalone serum is a specific response of the abalone-pathogen strain $V$. harveyi ORM4.

In line with these findings, we monitored the virulence of the $V$. harveyi ORM4 $\Delta l u x O$ mutant by comparing the survival rates of abalones following infection by either this 
mutant or the ORM4 wild type strain. V. harveyi ORM4 triggered a high mortality level, with $75 \%$ of death occurring within 10 days (Fig. 4). This mortality rate is similar to earlier results, confirms that $V$. harveyi ORM4 is a potent abalone pathogen, and that the abalones used in this study were mature and sensitive to $V$. harveyi ORM4 (Travers et al., 2009a; Travers et al., 2009b). Interestingly, when balneated with $V$. harveyi ORM4 $\Delta l u x O$, the survival rate of abalone drastically increased. This demonstrates that locking cells in a "high cell-density" -like state is detrimental for full virulence of $V$. harveyi ORM4. This is the exact opposite to observations recently reported for $V$. campbellii BAA-1116. In this strain, locking cells in a "maximally active QS" state (by the amino acid substitution D47A in Lux0) leads to a much lower survival rate of tiger grouper larvae (Epinephelus fuscoguttatus), as compared to larvae infected by the wild type strain. In contrast, locking cells to a "low cell-density" state (by the amino acid substitution D47E in Lux0) leads to an increased survival rate of these larvae (Noor et al., 2019).

QS is known to regulate virulence, notably through the regulation of secretion systems. Various secretion systems are thought to be involved in the pathogenicity of Vibrio toward molluscs, such as the T3SS in V. campbellii BAA-1116 (Ruwandeepika et al., 2015), or the type IV secretion system in Vibrio tapetis (Dias et al., 2018, Rahmani et al., in press). Interestingly, the genome of $V$. harveyi ORM4 harbours a complete set of T3SS genes (Fig. 5), but the role of the T3SS in the virulence of this strain and its QS-dependent regulation have never been addressed prior to this study. In V. campbellii BAA-1116, T3SS genes, such as exsA, encoding the major regulator of the T3SS (Zhou et al., 2008; Waters et al., 2010), or vopD, $v c r D$ and $v s c P$, encoding proteins involved in the structure and functioning of the T3SS, are repressed when cells are at "high cell-density"-like state (Waters et al., 2010; Ruwandeepika et al., 2015). To assess the link between QS and T3SS-mediated virulence in $V$. harveyi ORM4, we first monitored the expression of its exsA gene and compared it to its expression in the $\Delta l u x O$ background. Single-cell microscopy experiments showed that exsA is expressed in $V$. harveyi ORM4 under the tested conditions, and RT-qPCR confirmed that core T3SS-genes are expressed in this strain (Fig. 6). Moreover, expression of exsA is higher in early exponential phase, when cells are still at low cell density, as already demonstrated for V. campbellii BAA-1116 (Waters et al., 2010). However, the expression of exsA is also higher in the $\Delta l u x O$ mutant, as compared to the expression in the wild type, under all growth phases. This is rather surprising, given that this mutant is thought to be locked in a "high cell-density"-like state, while in $V$. 
campbellii BAA-1116, exsA expression is higher when cells are locked at low-cell density (Waters et al., 2010). Indeed, in the latter strain, LuxR directly represses exsA (Chaparian et al., 2020) by binding to its promoter, at both low- and high-cell densities (van Kessel et al., 2013). Our experiment shows that the regulation of the T3SS by QS is different between $V$. harveyi ORM4 and V. campbellii BAA-1116.

To further confirm that the T3SS is important for the virulence of $V$. harveyi ORM4, we subsequently constructed a derivative of $V$. harveyi ORM4 deficient in T3SS, by the deletion of the exsA gene. This strain has similar physiological characteristics as the wild type, and is still able to form biofilms (Fig. S5, S6 and S7), but fails to express T3SS genes (Fig. 6C). Importantly, when used in vivo by balneation with mature abalones, we observed that the $\triangle$ exs $A$ mutant is unable to induce mortality (Fig. 7A), while functional complementation restored the virulent phenotype in a similar level as the wild type one (Fig. 7B). These results demonstrate that the T3SS of $V$. harveyi ORM4 is essential for its virulence, similar to its role in V. parahaemolyticus (Zhou et al., 2010).

Taken together, our results suggest a different infection strategy between $V$. harveyi ORM4 and V. campbellii BAA-1116. Indeed, V. campbellii BAA-1116 is thought to have a so-called frontal attack strategy, where the expression of the T3SS operons of $V$. campbellii BAA-1116 is induced within 12 hours after infection of brine shrimps when cells are at low cell density, before expression decreases, at least one day before host death (Ruwandeepika et al., 2015). In contrast, $V$. harveyi ORM4 would express T3SS genes later in the process, when cells are at high-cell density, possibly after biofilm formation. In this scenario, locking cells at "high cell-density" -like state through the deletion of $\operatorname{luxO}$ is detrimental for ORM4 virulence, because of an incorrect timing of T3SS expression. Alternatively, the fact that the $\operatorname{lux} O$ mutant, despite overexpressing exsA, and an exs $A$ deletion mutant are both avirulent toward abalone might imply that another, but yetunknown, pathway is involved in the virulence. This pathway would be inactivated in the luxO mutant, rendering cells avirulent, independently of the presence of a functional T3SS. Genetic and transcriptomic comparisons between $V$. harveyi ORM4, the luxO mutant and the avirulent strain LMG 7890, as well as the physiological characterization of a double exsA-luxO deletion mutant will allow unveiling the genetic basis of virulence of $V$. harveyi ORM4 and its link with QS, and this will be the subject of a future work.

This article is protected by copyright. All rights reserved. 
In conclusion, the molecular mechanisms involved during infection of the European abalone $H$. tuberculata by $V$. harveyi ORM4 were investigated. Using genetic and functional approaches, we could demonstrate that the virulence of $V$. harveyi ORM4 is dependent on an intact QS system. This loss of virulence is correlated with a decreased motility and a lower ability to produce biofilm. Moreover, we could demonstrate that this loss of virulence is associated with a deregulation of the expression of the T3SS-regulator ExsA, and that the T3SS is essential to induce abalone mortality. Finally, we observed marked difference in phenotypes in the $\triangle l u x O$ mutant, as compared to what is known from the literature for V. campbellii BAA-1116 (formerly called V. harveyi (Lin et al., 2010)). Thus, previously-raised conclusions on the virulence mechanisms and strategy of $V$. harveyi strains using data from $V$. campbellii BAA-1116 may be over-extrapolated, and new $V$. harveyi model strains have to be established. With this study, we propose to use $V$. harveyi ORM4 as a new model strain to study the virulence mechanism of the $V$. harveyi species.

\section{Acknowledgments}

The authors would like to thank Frederique Le Roux for supplying strain E. coli $\beta-3914$. We also would like to thank the Genoscope for hosting the preliminary genome sequences of $V$. harveyi ORM4. Finally, we are grateful to Oceanopolis for the maintenance of abalones. This work was supported by the "Laboratoire d'Excellence" LabexMER (ANR10-LABX-19), by the "Ecole Universitaire de Recherche" ISblue (ANR-17-EURE-0015) and co-funded by a grant from the French government under the program "Investissements d'Avenir", and by a grant from the Regional Council of Brittany. This project received grants from the H2020 VIVALDI project (grant agreement ${ }^{\circ} 678589$ ), and the CNRS Prematuration (CoMAq project) and EC2CO (ViViMar project) programmes. The LBCM is supported by the Région Bretagne and European FEDER. AM and AJ are the recipient of doctoral fellowships co-funded by the Région Bretagne, France, and the Université de Bretagne-Sud.

\section{References}

Adler, J. (1966) Chemotaxis in bacteria. Science 153: 708-716. 
Anetzberger, C., Pirch, T., and Jung, K. (2009) Heterogeneity in quorum sensing-regulated bioluminescence of Vibrio harveyi. Mol Microbiol 73: 267-277.

Austin, B., and Zhang, X.H. (2006) Vibrio harveyi: a significant pathogen of marine vertebrates and invertebrates. Lett in Appl Microbiol 43: 119-124.

Ball, A.S., and van Kessel, J.C. (2019) The master quorum-sensing regulators LuxR/HapR directly interact with the alpha subunit of RNA polymerase to drive transcription activation in Vibrio harveyi and Vibrio cholerae. Mol Microbiol 111: 1317-1334.

Ball, A.S., Chaparian, R.R., and van Kessel, J.C. (2017) Quorum Sensing Gene Regulation by LuxR/HapR Master Regulators in Vibrios. J Bacteriol 199: e00105-17.

Bassis, C.M., and Visick, K.L. (2010) The cyclic-di-GMP phosphodiesterase BinA negatively regulates cellulose-containing biofilms in Vibrio fischeri. J Bacteriol 192: 1269-1278.

Bondad-Reantaso, M.G., Subasinghe, R.P., Arthur, J.R., Ogawa, K., Chinabut, S., Adlard, R. et al. (2005) Disease and health management in Asian aquaculture. Vet Parasitol 132: 249272.

Boyaci, H., Shah, T., Hurley, A., Kokona, B., Li, Z., Ventocilla, C. et al. (2016) Structure, Regulation, and Inhibition of the Quorum-Sensing Signal Integrator LuxO. PLoS Biol 14: e1002464.

Broberg, C.A., Zhang, L., Gonzalez, H., Laskowski-Arce, M.A., and Orth, K. (2010) A Vibrio effector protein is an inositol phosphatase and disrupts host cell membrane integrity. Science 329: 1660-1662.

Cardinaud, M., Offret, C., Huchette, S., Moraga, D., and Paillard, C. (2014a) The impacts of handling and air exposure on immune parameters, gene expression, and susceptibility to vibriosis of European abalone Haliotis tuberculata. Fish Shellfish Immunol 36: 1-8.

Cardinaud, M., Dheilly, N.M., Huchette, S., Moraga, D., and Paillard, C. (2015) The early stages of the immune response of the European abalone Haliotis tuberculata to a Vibrio harveyi infection. Dev Comp Immunol 51: 287-297.

Cardinaud, M., Barbou, A., Capitaine, C., Bidault, A., Dujon, A.M., Moraga, D., and Paillard, C. (2014b) Vibrio harveyi adheres to and penetrates tissues of the european abalone Haliotis tuberculata within the first hours of contact. Appl Environ Microbiol 80: 63286333.

Casillo, A., Lanzetta, R., Parrilli, M., and Corsaro, M.M. (2018) Exopolysaccharides from Marine and Marine Extremophilic Bacteria: Structures, Properties, Ecological Roles and Applications. Mar Drugs 16(2): 69

Chaparian, R.R., Ball, A.S., and van Kessel, J.C. (2020) Hierarchical Transcriptional Control of the LuxR Quorum-Sensing Regulon of Vibrio harveyi. J Bacteriol 202.

Delavat, F., Mitri, S., Pelet, S., and van der Meer, J.R. (2016) Highly variable individual donor cell fates characterize robust horizontal gene transfer of an integrative and conjugative element. Proc Natl Acad Sci U S A 113: E3375-E3383.

Delavat, F., Bidault, A., Pichereau, V., and Paillard, C. (2018) Rapid and efficient protocol to introduce exogenous DNA in Vibrio harveyi and Pseudoalteromonas sp. J Microbiol Methods 154: 1-5. 
Dias, G.M., Bidault, A., Le Chevalier, P., Choquet, G., Der Sarkissian, C., Orlando, L. et al. (2018) Vibrio tapetis Displays an Original Type IV Secretion System in Strains Pathogenic for Bivalve Molluscs. Front Microbiol 9: 227.

Dunn, A.K., Millikan, D.S., Adin, D.M., Bose, J.L., and Stabb, E.V. (2006) New rfp- and pES213-derived tools for analyzing symbiotic Vibrio fischeri reveal patterns of infection and lux expression in situ. Appl Environ Microbiol 72: 802-810.

Gode-Potratz, C.J., and McCarter, L.L. (2011) Quorum sensing and silencing in Vibrio parahaemolyticus. J Bacteriol 193: 4224-4237.

Gruenthal, K.M., and Burton, R.S. (2008) Genetic structure of natural populations of the California black abalone (Haliotis cracherodii Leach, 1814), a candidate for endangered species status. J Exp Mar Bio and Ecol 355: 47-58.

Harney, E., Dubief, B., Boudry, P., Basuyaux, O., Schilhabel, M.B., Huchette, S. et al. (2016) De novo assembly and annotation of the European abalone Haliotis tuberculata transcriptome. Mar Genomics 28: 11-16.

Herrera, A., and Satchell, K.J.F. (2020) Cross-Kingdom Activation of Vibrio Toxins by ADPRibosylation Factor Family GTPases. J Bacteriol 202.

Hobday, A.J., Tegner, M.J., and Haaker, P.L. (2001) Over-exploitation of a broadcast spawning marine invertebrate: Decline of the white abalone. Rev Fish Biol Fish 10: 493514.

Huchette, S.M.H., and Clavier, J. (2004) Status of the ormer (Haliotis tuberculata L.) industry in Europe. J Shellfish Res 23: 951-955.

Hueck, C.J. (1998) Type III protein secretion systems in bacterial pathogens of animals and plants. Microbiol Mol Biol Rev 62: 379-433.

Ina-Salwany, M.Y., Al-Saari, N., Mohamad, A., Mursidi, F.A., Mohd-Aris, A., Amal, M.N.A. et al. (2019) Vibriosis in Fish: A Review on Disease Development and Prevention. J Aquat Anim Health 31: 3-22.

Lauriano, C.M., Ghosh, C., Correa, N.E., and Klose, K.E. (2004) The sodium-driven flagellar motor controls exopolysaccharide expression in Vibrio cholerae. J Bacteriol 186: 48644874.

Le Roux, F. (2017) Environmental vibrios: "a walk on the wild side". Environ Microbiol Rep 9: 27-29.

Le Roux, F., Binesse, J., Saulnier, D., and Mazel, D. (2007) Construction of a Vibrio splendidus mutant lacking the metalloprotease gene vsm by use of a novel counterselectable suicide vector. Appl Environ Microbiol 73: 777-784.

Lee, K.K., Liu, P.C., and Chuang, W.H. (2002) Pathogenesis of gastroenteritis caused by Vibrio carchariae in cultured marine fish. Mar Biotechnol (NY) 4: 267-277.

Lin, B.C., Wang, Z., Malanoski, A.P., O'Grady, E.A., Wimpee, C.F., Vuddhakul, V. et al. (2010) Comparative genomic analyses identify the Vibrio harveyi genome sequenced strains BAA-1116 and HY01 as Vibrio campbellii. Environ Microbiol Rep 2: 81-89.

Livak, K.J., and Schmittgen, T.D. (2001) Analysis of relative gene expression data using real-time quantitative PCR and the 2(-Delta Delta C(T)) Method. Methods 25: 402-408.

This article is protected by copyright. All rights reserved. 
Lorenz, N., Shin, J.Y., and Jung, K. (2017) Activity, Abundance, and Localization of Quorum Sensing Receptors in Vibrio harveyi. Front Microbiol 8: 634.

Luo, P., He, X.Y., Liu, Q.T., and Hu, C.Q. (2015) Developing Universal Genetic Tools for Rapid and Efficient Deletion Mutation in Vibrio Species Based on Suicide T-Vectors Carrying a Novel Counterselectable Marker, vmi480. Plos One 10: e0144465

Miller, M.B., and Bassler, B.L. (2001) Quorum sensing in bacteria. Annu Rev Microbiol 55: 165-199.

Milton, D.L. (2006) Quorum sensing in vibrios: complexity for diversification. Int J Med Microbiol 296: 61-71.

Miyamoto, C.M., Dunlap, P.V., Ruby, E.G., and Meighen, E.A. (2003) LuxO controls luxR expression in Vibrio harveyi: evidence for a common regulatory mechanism in Vibrio. $\mathrm{Mol}$ Microbiol 48: 537-548.

Miyashiro, T., and Ruby, E.G. (2012) Shedding light on bioluminescence regulation in Vibrio fischeri. Mol Microbiol 84: 795-806.

Montanchez, I., and Kaberdin, V.R. (2020) Vibrio harveyi: A brief survey of general characteristics and recent epidemiological traits associated with climate change. Mar Environ Res 154: 104850.

Nackerdien, Z.E., Keynan, A., Bassler, B.L., Lederberg, J., and Thaler, D.S. (2008) Quorum sensing influences Vibrio harveyi growth rates in a manner not fully accounted for by the marker effect of bioluminescence. PLoS One 3: e1671.

Nealson, K.H., Platt, T., and Hastings, J.W. (1970) Cellular control of the synthesis and activity of the bacterial luminescent system.J Bacteriol 104: 313-322.

Ng, W.L., and Bassler, B.L. (2009) Bacterial quorum-sensing network architectures. Annu Rev Genet 43: 197-222.

Nicolas, J.L., Basuyaux, O., Mazurie, J., and Thebault, A. (2002) Vibrio carchariae, a pathogen of the abalone Haliotis tuberculata. Dis Aquat Organ 50: 35-43.

Noor, N.M., Defoirdt, T., Alipiah, N., Karim, M., Daud, H., and Natrah, I. (2019) Quorum sensing is required for full virulence of Vibrio campbellii towards tiger grouper (Epinephelus fuscoguttatus) larvae. J Fish Dis 42: 489-495.

Paillard, C., Le Roux, F., and Borrego, J.J. (2004) Bacterial disease in marine bivalves, a review of recent studies: Trends and evolution. Aquat Living Resour 17: 477-498.

Plano, G.V., and Schesser, K. (2013) The Yersinia pestis type III secretion system: expression, assembly and role in the evasion of host defenses. Immunol Res 57: 237-245.

Rahmani, A., Delavat, F., Lambert, C., Le Goic, N., Dabas, E., Paillard, C., and Pichereau, V. Implication of the type IV secretion system in the pathogenicity of Vibrio tapetis, the etiological agent of Brown Ring Disease affecting the Manila clam Ruditapes philippinarum. Front Cell Infect Microbiol. in press

Ruwandeepika, H.A.D., Karunasagar, I., Bossier, P., and Defoirdt, T. (2015) Expression and Quorum Sensing Regulation of Type III Secretion System Genes of Vibrio harveyi during Infection of Gnotobiotic Brine Shrimp. Plos One 10: e0143935 
Ruwandeepika, H.A.D., Jayaweera, T.S.P., Bhowmick, P.P., Karunasagar, I., Bossier, P., and Defoirdt, T. (2012) Pathogenesis, virulence factors and virulence regulation of vibrios belonging to the Harveyi clade. Reviews Aquacult 4: 59-74.

Sreelatha, A., Bennett, T.L., Zheng, H., Jiang, Q.X., Orth, K., and Starai, V.J. (2013) Vibrio effector protein, VopQ, forms a lysosomal gated channel that disrupts host ion homeostasis and autophagic flux. Proc Natl Acad Sci U S A 110: 11559-11564.

Tischler, A.H., Lie, L., Thompson, C.M., and Visick, K.L. (2018) Discovery of Calcium as a Biofilm-Promoting Signal for Vibrio fischeri Reveals New Phenotypes and Underlying Regulatory Complexity. J Bacteriol 200.

Travers, M.A., Boettcher Miller, K., Roque, A., and Friedman, C.S. (2015) Bacterial diseases in marine bivalves. J Invertebr Pathol 131: 11-31.

Travers, M.A., BArbou, A., Le Goic, N., Huchette, S., Paillard, C., and Koken, M. (2008) Construction of a stable GFP-tagged Vibrio harveyi strain for bacterial dynamics analysis of abalone infection. FEMS Microbiol Lett 289: 34-40.

Travers, M.A., Basuyaux, O., Le Goic, N., Huchette, S., Nicolas, J.L., Koken, M., and Paillard, C. (2009a) Influence of temperature and spawning effort on Haliotis tuberculata mortalities caused by Vibrio harveyi: an example of emerging vibriosis linked to global warming. Glob Chang Biol 15: 1365-1376.

Travers, M.A., Le Bouffant, R., Friedman, C.S., Buzin, F., Cougard, B., Huchette, S. et al. (2009b) Pathogenic Vibrio harveyi, in contrast to non-pathogenic strains, intervenes with the p38 MAPK pathway to avoid an abalone haemocyte immune response. J Cell Biochem 106: $152-160$.

Tu, K.C., and Bassler, B.L. (2007) Multiple small RNAs act additively to integrate sensory information and control quorum sensing in Vibrio harveyi. Genes Dev 21: 221-233.

van Kessel, J.C., Rutherford, S.T., Shao, Y., Utria, A.F., and Bassler, B.L. (2013) Individual and Combined Roles of the Master Regulators AphA and LuxR in Control of the Vibrio harveyi Quorum-Sensing Regulon. J Bacteriol 195: 436-443.

Vandenberghe, J., Verdonck, L., Robles-Arozarena, R., Rivera, G., Bolland, A., Balladares, M. et al. (1999) Vibrios associated with Litopenaeus vannamei larvae, postlarvae, broodstock, and hatchery probionts. Appl Environ Microbiol 65: 2592-2597.

Waddell, B., Southward, C.M., McKenna, N., and Devinney, R. (2014) Identification of VPA0451 as the specific chaperone for the Vibrio parahaemolyticus chromosome 1 type III-secreted effector VPA0450. FEMS Microbiol Lett 353: 141-150.

Waters, C.M., Wu, J.T., Ramsey, M.E., Harris, R.C., and Bassler, B.L. (2010) Control of the Type 3 Secretion System in Vibrio harveyi by Quorum Sensing through Repression of ExsA. Appl Environ Microbiol 76: 4996-5004.

Watnick, P.I., and Kolter, R. (1999) Steps in the development of a Vibrio cholerae El Tor biofilm. Mol Microbiol 34: 586-595.

Yang, Q., and Defoirdt, T. (2015) Quorum sensing positively regulates flagellar motility in pathogenic Vibrio harveyi. Environ Microbiol 17: 960-968. 
Yip, E.S., Grublesky, B.T., Hussa, E.A., and Visick, K.L. (2005) A novel, conserved cluster of genes promotes symbiotic colonization and sigma-dependent biofilm formation by Vibrio fischeri. Mol Microbiol 57: 1485-1498.

Zhou, X.H., Konkel, M.E., and Call, D.R. (2010) Regulation of type III secretion system 1 gene expression in Vibrio parahaemolyticus is dependent on interactions between ExsA, ExsC and ExsD. Virulence 1: 260-272.

Zhou, X.H., Shah, D.H., Konkel, M.E., and Call, D.R. (2008) Type III secretion system 1 genes in Vibrio parahaemolyticus are positively regulated by ExsA and negatively regulated by ExsD. Mol Microbiol 69: 747-764.

Zhu, J., and Mekalanos, J.J. (2003) Quorum sensing-dependent biofilms enhance colonization in Vibrio cholerae. Dev Cell 5: 647-656.

Zhu, J., Miller, M.B., Vance, R.E., Dziejman, M., Bassler, B.L., and Mekalanos, J.J. (2002) Quorum-sensing regulators control virulence gene expression in Vibrio cholerae. Proc Natl Acad Sci U S A 99: 3129-3134. 


\section{Figure legends}

Fig. 1. Doubling times of V. harveyi ORM4 and V. harveyi ORM4 $\Delta l u x O$. ORM4 (strain 1) and ORM4 $\Delta l u x O$ (strain 243) were grown in erlenmeyers with shaking at the indicated temperature, and $\mathrm{OD}_{600 \mathrm{~nm}}$ was measured regularly to calculate the doubling time. An unpaired two-tailed t-test was performed for each temperature.

Fig. 2. Motility of $\boldsymbol{V}$. harveyi ORM4 and V. harveyi ORM4 $\Delta l u x O$. ORM4 (strain 1) and ORM4 $\Delta$ luxO (strain 243) were spotted on $0.2 \%$ agar plates, and swimming diameters were recorded every two hours, both at $20^{\circ} \mathrm{C}(\mathrm{A})$ and $28^{\circ} \mathrm{C}(\mathrm{B})$. Note that the swimming diameter at $24 \mathrm{~h}$ could not be recorded at $28^{\circ} \mathrm{C}(\mathrm{B})$, because the halos overlapped, and that no expansion of the colony diameter due to swimming was observed before $6 \mathrm{~h}(\mathrm{~A})$ and $4 \mathrm{~h}(\mathrm{~B})$. Unpaired two-tailed t-tests with equal variance were performed to compare $V$. harveyi ORM4 with the $\Delta l u x O$ mutant for every condition, except for the condition LBS at $8 \mathrm{~h}$ at $20^{\circ} \mathrm{C}$ (non-parametric, Wilcoxon test).

Fig. 3. Biofilm production by V. harveyi ORM4, V. harveyi ORM4 $\Delta l u x O$ and V. harveyi LMG 7890. Strains ORM4 (strain 242), ORM4 $\Delta l u x O$ (strain 267) and LMG 7890 (strain 263) were grown in LBS (A and B) and in abalone serum (C and D), both at $20^{\circ} \mathrm{C}(\mathrm{A}$ and C) and $28^{\circ} \mathrm{C}$ (B and D) without shaking in microplates, and biofilm was stained after 48 hours using crystal violet. (E) and (F) show the media effect (LBS vs. serum) for all three strains, at $20^{\circ} \mathrm{C}$ in (E) and $28^{\circ} \mathrm{C}$ in (F). A one-way ANOVA (with Tukey test) was performed for every condition in panels $A$ to $D$, and an unpaired two-tailed t-test was performed for to compare the media effect in panels $\mathrm{E}$ and $\mathrm{F}$.

Fig. 4. Survival rate of abalones in the presence of $V$. harveyi ORM4, V. harveyi ORM4 AluxO and V. harveyi LMG 7890. In (A), ORM4 (strain 1), LMG 7890 (strain 45) and ORM4 $\Delta l u x O$ (strain 243) were used to infect abalones by balneation, and surviving abalones were counted daily. Every experiment was performed in triplicate tanks, but one replicate for the $\operatorname{lux} O$ mutant was removed because of a bubbling problem. Note that purple (non-infected tanks) and orange (tanks infected with $V$. harveyi LMG 7890) curves overlap. Note that the results for ORM4, LMG 7890 and non-infected tanks are identical 
to the ones in Fig. 7A, because experiments were performed at the same time. In (B), strains 256 (ORM4), $306(\Delta l u x O)$ and 272 ( $\Delta$ luxO compl-luxO) were used for abalone infections by balneation. Note that the results for ORM4 and non-infected tanks are identical to the ones in Fig. 4B, because experiments were performed at the same time. A two-way ANOVA (with Tukey test) was performed for every condition, and $p$ values are relative to non-infected abalones. +/- was calculated using standard deviation.

Fig. 5. Comparison of the genetic organisations of the T3SS regions between $V$. harveyi ORM4 and V. parahaemolyticus RIMD2210633. MAGE Genoscope (https://mage.genoscope.cns.fr/microscope/home/index.php?) was used to annotate the T3SS genes in $V$. harveyi ORM4 and to calculate the amino acid identity percentage between proteins of the two bacteria. Genes are classified according to the type of proteins produced: structure (yellow), regulator (blue), ATPase (green), effector (red), and non-identified (grey). In the T3SS1 loci of V. parahaemolyticus RIMD2210633, a region located between genes $v s c U$ and $v s c L$ is composed of genes encoding for VopQ (vp1680), VopR (vp1683), VopS (vp1686), and hypothetical proteins that could be potential effectors. Genes encoding proteins with amino acid identity $>50 \%$ and query coverage $>50 \%$ are linked with a full line and genes encoding proteins with amino acid identity between $30 \%$ and $50 \%$ and a query coverage $>50 \%$ are linked with a dotted line. The percentage of amino acid identity between $V$. harveyi ORM4 and $V$. parahaemolyticus RIMD2210633 for every gene of the T3SS, and their predicted function, can be found in Table S5, together with a previously proposed unified nomenclature (Hueck, 1998).

Fig. 6. Regulation of T3SS genes in $V$. harveyi ORM4 and derivative mutants. (A), GFP fluorescence produced in ORM4 (strain 249) and ORM4 $\Delta l u x O$ (strain 332) from the PexsA$g f p$ transcriptional fusion, quantified at different growth phases (3h: early exponentialphase. 6h: mid-exponential phase. $9 \mathrm{~h}$ : late exponential-phase. $0 / \mathrm{N}$ : Overnight, stationary phase). An unpaired two-tailed t-test with equal SD was performed to compare the fluorescence from both genetic backgrounds at each time point. (B), illustration of the higher fluorescence of GFP emitted from the $\triangle l u x O$ background. Images were obtained at time 6 hours and scaled to the same maximal Average Grey Value (found in the image from the $\triangle l u x O$ background). (C), RT-qPCR experiments on targeted T3SS genes, when cells are at low-cell density $\left(\mathrm{OD}_{600 \mathrm{~nm}} 0.1\right)$. White histograms correspond to $V$. harveyi 
ORM4, while pale and and dark grey ones correspond to the $\triangle e x s A$ and $\triangle l u x O$ mutants, respectively. HORM4v2_240099 encodes a T3SS chaperone, YscB family; HORM4v2_240127 encodes the ATPase VscN. A multiple-comparison two-way ANOVA (with Tukey test) was performed for both genes, using the $\log _{2}$ values of the $2^{-\triangle \Delta C T}$ method.

Fig. 7. Survival rate of abalones in the presence of $V$. harveyi ORM4, V. harveyi ORM4 $\Delta$ exs $\boldsymbol{A}$ and $\boldsymbol{V}$. harveyi LMG 7890. In (A), ORM4 (strain 1), (LMG 7890 (strain 45) and ORM4 $\Delta$ exsA (strain 131) were used to infect abalones by balneation, and surviving abalones were counted daily. Every experiment was performed in triplicate tanks, but one replicate for the exsA mutant was removed because of a bubbling problem in one replicate. Note that purple (Non-infected tanks), orange (tanks infected with $V$. harveyi LMG 7890) and blue (tanks infected with $V$. harveyi ORM4 $\Delta$ exs $A$ ) curves overlap. Note that the results for ORM4, LMG 7890 and non-infected tanks are identical to the ones in Fig. 4A, because experiments were performed at the same time. In (B), ORM4 (strain 256), ORM4 $\Delta$ exs $A$ (strain 293) and ORM4 $\Delta$ exsA compl-exsA (strain 162) were used for abalone infections by balneation. Note that the results for ORM4 and non-infected tanks are identical to the ones in Fig. 4B, because experiments were performed at the same time. A two-way ANOVA (with Tukey test) was performed for every condition, and $p$ values are relative to noninfected abalones. +/- was calculated using standard deviation. 
Fig. S1. Doubling time of $V$. harveyi ORM4, ORM4 $\triangle l u x O$, and complemented ORM4 SluxO. ORM4 (strain 256), ORM4 $\Delta l u x O$ (strain 306) and ORM4 $\Delta l u x O$ compl-luxO (strain 272) were grown in erlenmeyers with shaking, and $\mathrm{OD}_{600 \mathrm{~nm}}$ was measured regularly to calculate the doubling time. A one-way ANOVA (with Tukey test) was performed for every condition.

Fig. S2. Motility of $V$. harveyi ORM4, ORM4 $\Delta l u x O$, and complemented ORM4 $\Delta l u x O$. ORM4 (strain 256), ORM4 $\Delta l u x O$ (strain 306) and ORM4 $\Delta l u x O$ compl-luxO (strain 272) were spotted on $0.2 \%$ agar plates, and swimming diameter was recorded after 10 hours at $20^{\circ} \mathrm{C}(\mathrm{A}$ and $\mathrm{B})$ and $28^{\circ} \mathrm{C}(\mathrm{C}$ and $\mathrm{D})$, on LBS (A and C) and "poor" (B and D) swimming plates. A one-way ANOVA (with Tukey test) was performed for every condition.

Fig. S3. Biofilm production by $\boldsymbol{V}$. harveyi strains. ORM4 (strain 256), ORM4 $\Delta l u x O$ (strain 306), ORM4 $\Delta l u x O$ compl-luxO (strain 272) and LMG 7890 (strain 283) were grown at $28^{\circ} \mathrm{C}$, both in LBS (A) and in abalone serum (B) without shaking in microplates, and biofilm was stained after 24 hours using crystal violet. A one-way ANOVA (with Tukey test) was performed for every condition.

Fig. S4. Exopolysaccharide production of V. harveyi ORM4, ORM4 $\Delta l u x O$, and complemented ORM4 $\Delta$ luxO. ORM4 (strain 256), ORM4 $\Delta l u x O$ (strain 306) and ORM4 $\Delta l u x O$ compl-luxO (strain 272) were spotted on LBS+Trim plates containing Congo Red, and incubated for 24 hours.

Fig. S5. Doubling time of V. harveyi ORM4 and V. harveyi ORM4 $\Delta$ exsA. ORM4 (strain 1) and ORM4 $\triangle$ exsA (strain 131) were grown in erlenmeyers with shaking, and $0 D_{600 \mathrm{~nm}}$ was measured regularly to calculate the doubling time. Note that the result for ORM4 is identical to the one in Fig. 1, because experiments were performed at the same time. An unpaired two-tailed t-test has been performed for each temperature.

Fig. S6. Motility of $V$. harveyi ORM4, ORM4 $\Delta$ exs A, and complemented ORM4 $\Delta$ exs $A$. ORM4 (strain 256), ORM4 $\Delta$ exsA (strain 293) and ORM4 $\Delta$ exsA compl-exsA (strain 162) were spotted on $0.2 \%$ agar plates, and swimming diameter was recorded after 10 hours, both at $20^{\circ} \mathrm{C}(\mathrm{A}$ and $\mathrm{B})$ and $28^{\circ} \mathrm{C}(\mathrm{C}$ and $\mathrm{D})$, both on LBS (A and $\left.\mathrm{C}\right)$ and "poor" (B and $\left.\mathrm{D}\right)$ 
swimming plates. A one-way ANOVA (with Tukey test) was performed for every condition.

Fig. S7. Biofilm production by $V$. harveyi ORM4, V. harveyi ORM4 $\Delta$ exsA and $V$. harveyi LMG 7890. ORM4 (strain 242), ORM4 $\Delta$ exsA (strain 264) and LMG 7890 (strain 263) were grown at $20^{\circ} \mathrm{C}(\mathrm{A}$ and $\mathrm{B})$ and $28^{\circ} \mathrm{C}(\mathrm{C}$ and $\mathrm{D})$, both in LBS (A and C) and in abalone serum (B and D) without shaking in microplates, and biofilm was stained after 24 hours using crystal violet. Note that the results for ORM4 and LMG 7890 are identical to the ones in Fig. 3, because experiments were performed at the same time. A one-way ANOVA (with Tukey test) was performed for every condition. 
$20^{\circ} \mathrm{C}$

$28^{\circ} \mathrm{C}$

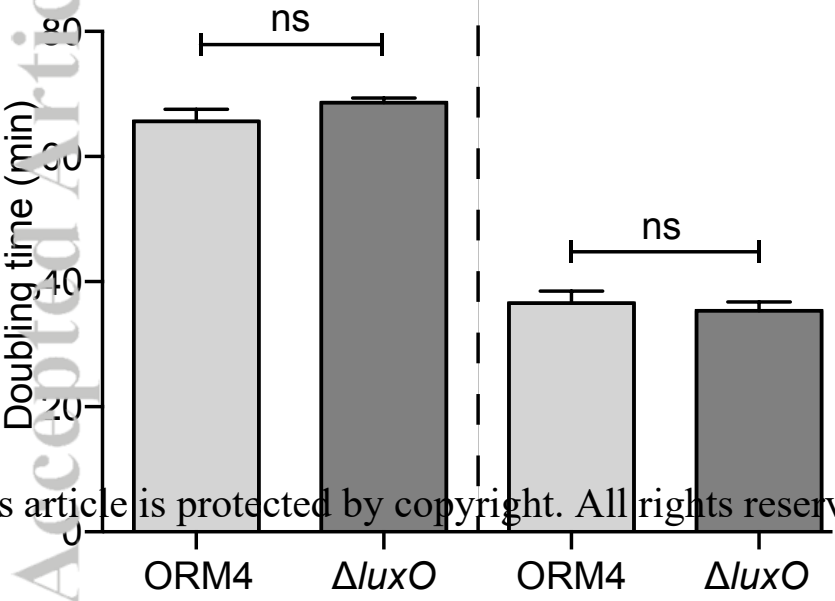




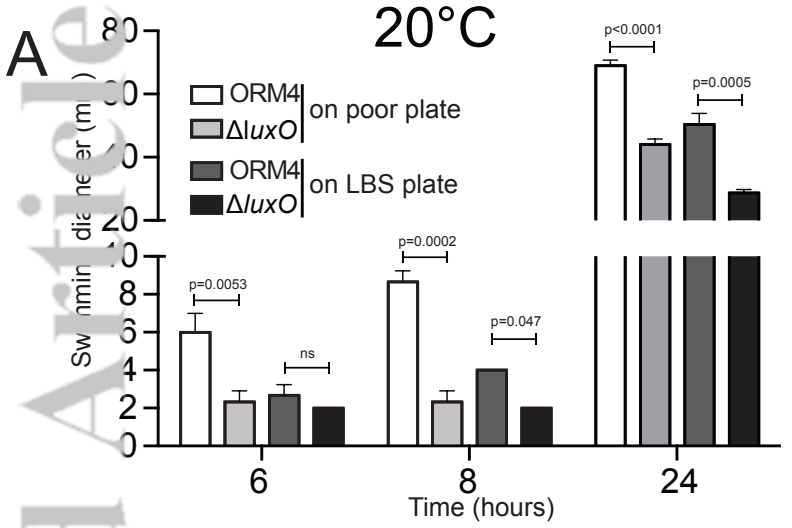




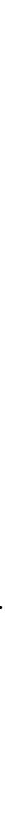



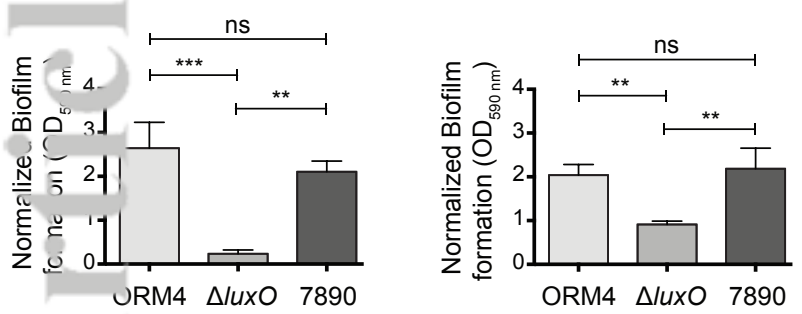

$20^{\circ} \mathrm{C}$-serum

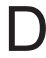

\section{$28^{\circ} \mathrm{C}$-serum}

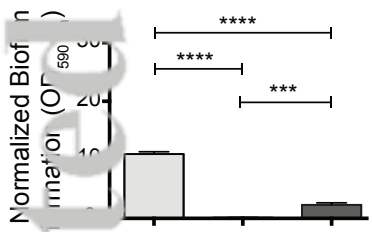

ORM4 $\Delta / u x O 7890$
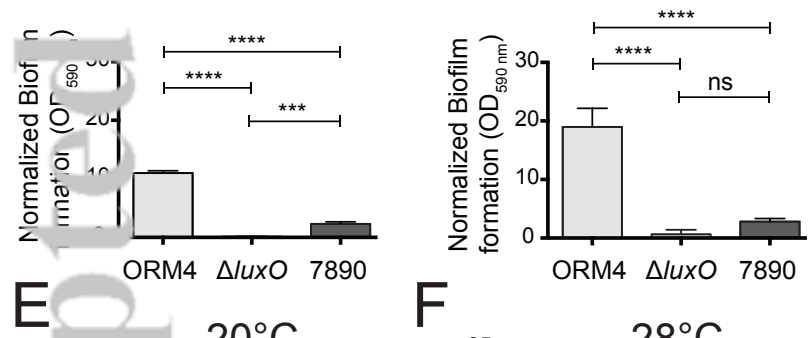

ORM4 $\Delta / u x O 7890$

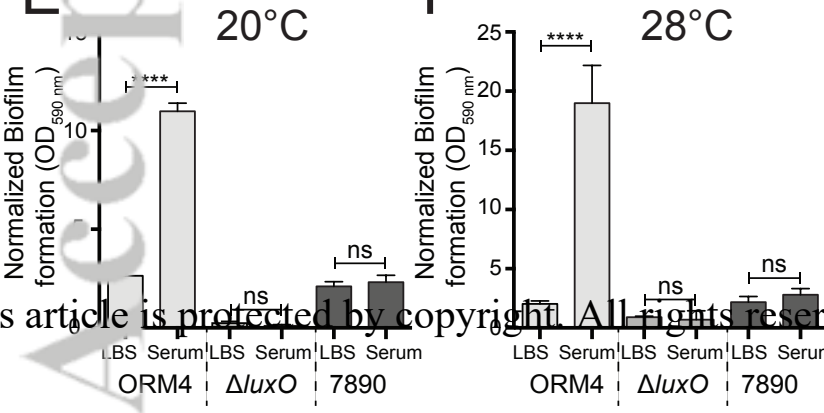



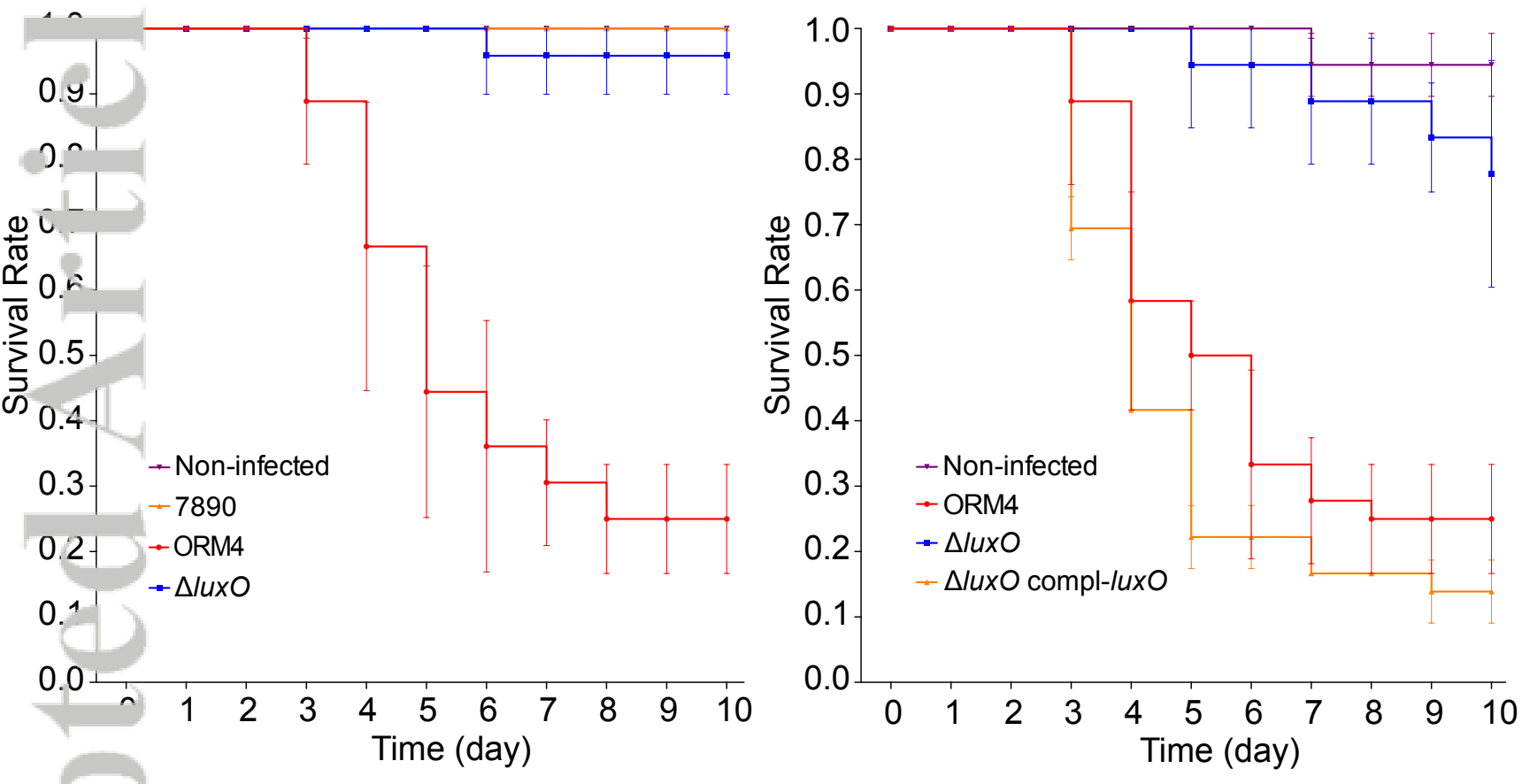

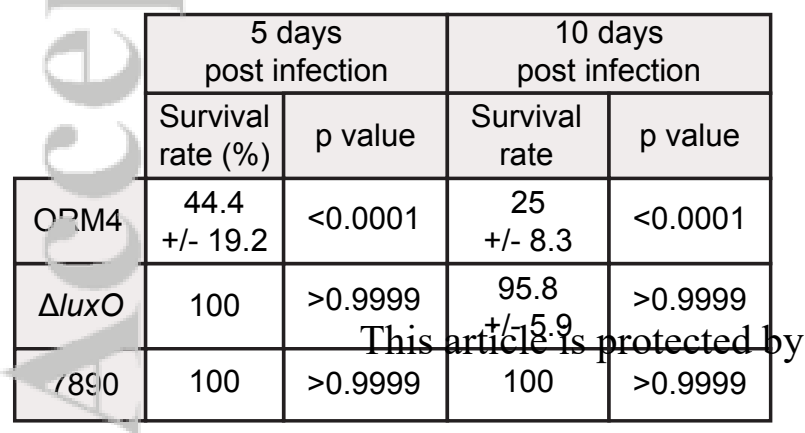

\begin{tabular}{|c|c|c|c|c|}
\hline & \multicolumn{2}{|c|}{$\begin{array}{c}5 \text { days } \\
\text { post infection }\end{array}$} & \multicolumn{2}{|c|}{$\begin{array}{c}10 \text { days } \\
\text { post infection }\end{array}$} \\
\hline & $\begin{array}{l}\text { Survival } \\
\text { rate (\%) }\end{array}$ & $p$ value & $\begin{array}{l}\text { Survival } \\
\text { rate }\end{array}$ & $p$ value \\
\hline ORM4 & $\begin{array}{c}50 \\
+/-8.3\end{array}$ & $<0.0001$ & $\begin{array}{c}25 \\
+/-8.3\end{array}$ & $<0.0001$ \\
\hline $\begin{array}{c}\Delta / u x O \\
\text { byright. A }\end{array}$ & $\begin{array}{c}94.4 \\
\text { +r-og. } \\
\text { riotits }\end{array}$ & $\begin{array}{c}0.7397 \\
\text { ceserved. }\end{array}$ & $\begin{array}{r}77.7 \\
+/-17.3 \\
\end{array}$ & 0.0156 \\
\hline $\begin{array}{l}\Delta \text { compl-luxO } \\
\text { com }\end{array}$ & $\begin{array}{r}22.2 \\
+/-4.8 \\
\end{array}$ & & $\begin{array}{r}13.9 \\
+/-4.8 \\
\end{array}$ & $<0.0001$ \\
\hline
\end{tabular}




\section{$A$}

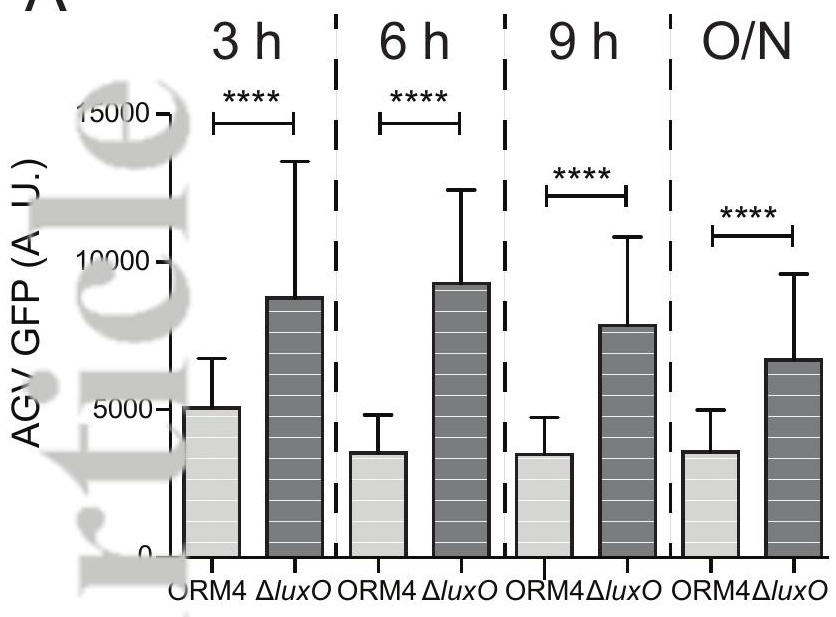

\section{B}

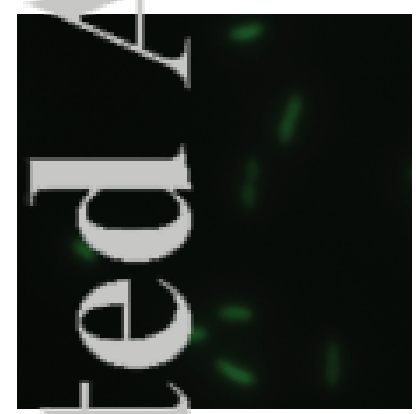

URM4

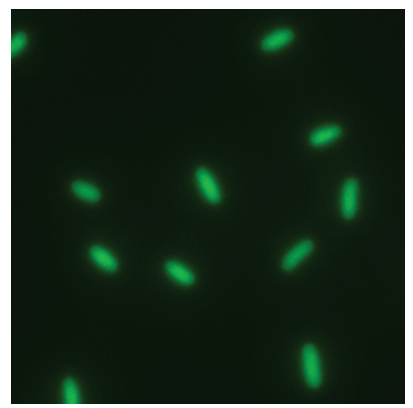

$\Delta / u x O$

C

M4v2_240099 | HORM4v2_240127

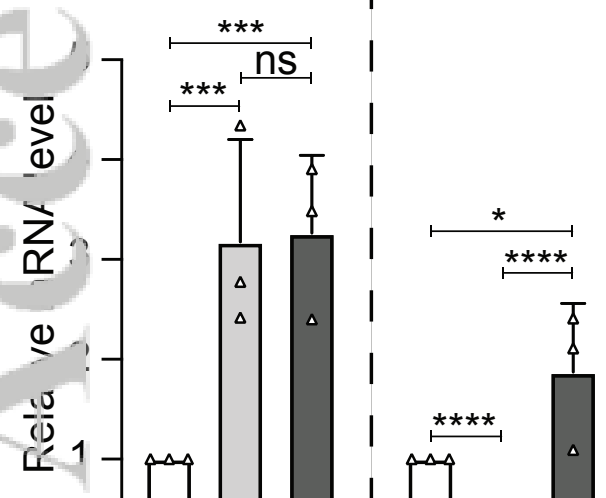

s article is protected by copyriqht. Adl rights reserv

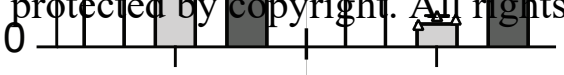



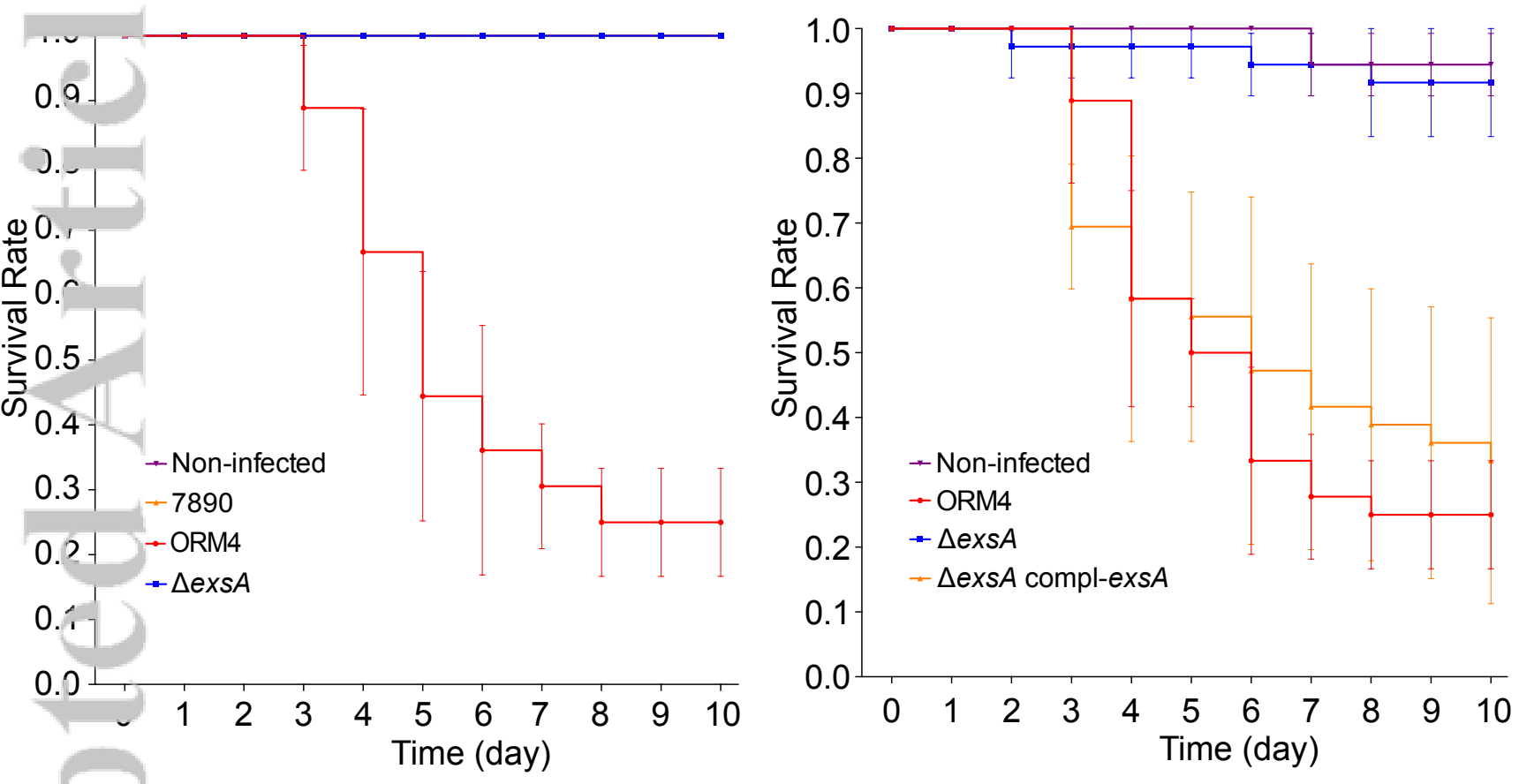

\begin{tabular}{|c|c|c|c|c|}
\cline { 2 - 5 } \multicolumn{1}{c|}{} & \multicolumn{2}{c|}{$\begin{array}{c}5 \text { days } \\
\text { post infection }\end{array}$} & \multicolumn{2}{c|}{$\begin{array}{c}\text { days } \\
\text { post infection }\end{array}$} \\
\cline { 2 - 5 } & $\begin{array}{c}\text { Survival } \\
\text { rate (\%) }\end{array}$ & $\mathrm{p}$ value & $\begin{array}{c}\text { Survival } \\
\text { rate }\end{array}$ & $\mathrm{p}$ value \\
\hline PRM4 & $\begin{array}{c}44.4 \\
+/-19.2\end{array}$ & $<0.0001$ & $\begin{array}{c}25 \\
+/-8.3\end{array}$ & $<0.0001$ \\
\hline SexsA & 100 & $\begin{array}{c}>0.9999 \\
\text { This anticle is protected by } \\
100\end{array}$ & $>0.9999$ \\
\hline 7390 & 100 & $>0.9999$ & 100 & $>0.9999$ \\
\hline
\end{tabular}

\begin{tabular}{|c|c|c|c|c|}
\cline { 2 - 5 } \multicolumn{1}{c|}{} & \multicolumn{2}{c|}{$\begin{array}{c}5 \text { days } \\
\text { post infection }\end{array}$} & \multicolumn{2}{c|}{$\begin{array}{c}\text { days } \\
\text { post infection }\end{array}$} \\
\cline { 2 - 5 } \multicolumn{1}{c|}{} & $\begin{array}{c}\text { Survival } \\
\text { rate (\%) }\end{array}$ & p value & $\begin{array}{c}\text { Survival } \\
\text { rate }\end{array}$ & p value \\
\hline ORM4 & $\begin{array}{c}50 \\
+/-8.3\end{array}$ & $<0.0001$ & $\begin{array}{c}25 \\
+/-8.3\end{array}$ & $<0.0001$ \\
\hline $\begin{array}{c}\Delta \text { exsA } \\
\text { copright. }\end{array}$ & $\begin{array}{c}97.2 \\
1+/-4.8\end{array}$ & 0.9886 & $\begin{array}{c}91.7 \\
+/-8.3\end{array}$ & 0.9886 \\
\begin{tabular}{c}
\hline rexs $A+$ \\
compl-exsA
\end{tabular} & $\begin{array}{c}55.6 \\
+/-19.2\end{array}$ & $<0.0001$ & $\begin{array}{c}33.3 \\
+/-22.0\end{array}$ & $<0.0001$ \\
\hline
\end{tabular}

